\title{
Cavity control of a single-electron quantum cyclotron: Measuring the electron magnetic moment
}

\author{
D. Hanneke, ${ }^{*}$ S. Fogwell Hoogerheide, and G. Gabrielse ${ }^{\dagger}$ \\ Department of Physics, Harvard University, Cambridge, Massachusetts 02138, USA
}

(Received 3 September 2010; revised manuscript received 24 January 2011; published 24 May 2011)

\begin{abstract}
Measurements with a one-electron quantum cyclotron determine the electron magnetic moment, given by $g / 2=1.00115965218073(28)\left[0.28 \mathrm{ppt}\right.$, and the fine structure constant, $\alpha^{-1}=137.035999084(51)$ [0.37ppb]. Announcements of these measurements [Phys. Rev. Lett. 97, 030801 (2006); 100, 120801 (2008)] are supplemented here with a more complete description of the one-electron quantum cyclotron and the measurement methods, a discussion of the cavity control of the radiation field, a summary of the analysis of the measurements, and a fuller discussion of the uncertainties.
\end{abstract}

\section{INTRODUCTION}

\section{A. The electron magnetic moment}

Measurements of the electron magnetic moment $\boldsymbol{\mu}$ probe the interaction of the electron with the fluctuating vacuum, allow the highest accuracy determination of the fine structure constant, and sensitively test quantum electrodynamics (QED). For an eigenstate of spin $\mathbf{S}$,

$$
\boldsymbol{\mu}=-\frac{g}{2} \mu_{B} \frac{\mathbf{S}}{\hbar / 2},
$$

where $g / 2$ is the magnitude of $\boldsymbol{\mu}$ scaled by the Bohr magneton $\mu_{B}=e \hbar /(2 m)$.

For angular momentum arising from orbital motion, $g / 2$ depends on the relative distribution of charge and mass and equals $1 / 2$ if they coincide, for example cyclotron motion in a magnetic field. For a point particle in a renormalizable Dirac description, $g / 2=1$, and deviations from this value probe a particle's interactions with the vacuum as well as the nature of the particle itself, as with the proton whose $g / 2 \approx 2.8$ arises from its quark-gluon composition.

\section{B. New measurements of the electron moment}

Our new measurements, announced in 2006 [1] and 2008 [2], used a one-electron quantum cyclotron [3] to determine the electron $g / 2$ to a $0.76 \mathrm{ppt}$ and then to a 0.28 ppt accuracy. The latter result,

$$
g / 2=1.00115965218073(28) \quad[0.28 \mathrm{ppt}],
$$

has an uncertainty that is 2.7 and 15 times smaller than the 2006 and 1987 measurements (Fig. 1), the latter being a measurement that stood for nearly 20 years [4]. The electron $g$ is measured with an uncertainty that is 2300 times smaller than has been achieved for the heavier muon lepton [5].

The central feature of the new measurements is the quantum jump spectroscopy of completely resolved cyclotron and spin levels of a one-electron quantum cyclotron [3]. A number of new methods were introduced to make this possible.

(1) A cylindrical Penning trap cavity that was invented for these experiments [6] imposes boundary conditions upon the

\footnotetext{
*Current address: Amherst College, Amherst, MA 01002.

† gabrielse@physics.harvard.edu
}

radiation field as well as providing an electrostatic quadrupole potential in which a single particle can be suspended and observed [7].

(2) The resulting cavity-inhibited spontaneous emission, at a rate 10 to 50 times below the radiation rate in free space, gives the averaging time required to resolve one-quantum transitions that are made when all detection systems are turned off.

(3) Stored electron plasmas [8-10] and the damping of a single electron in this cavity [2] are used together to determine cavity frequency shifts and eliminate cavity shifts as a major uncertainty [2].

(4) Blackbody photons that would cause unwanted quantum jumps are eliminated by lowering the cavity temperature to $100 \mathrm{mK}$ with a dilution refrigerator [3].

(5) Quantum nondemolition measurements of the cyclotron and spin energy level are realized using a one-particle self-excited oscillator [11].

(6) The stored electron serves as its own magnetometer, allowing the accumulation of lineshape statistics over days, revealing that a broadening of the expected lineshapes is the major remaining uncertainty [2].

Following sections will discuss the quantum cyclotron and the new methods. Still further details appear in a thesis [12].

\section{A long history}

As befits one of the few properties of the electron that can be accurately measured, the Harvard magnetic moment measurements detailed here are only the latest in a long history of measurements that make use of different methods. The early history [13] established that $g / 2 \approx 1$. A series of more precise measurements followed at the University of Michigan, by measuring the difference of the cyclotron and spin precession frequencies of $\mathrm{keV}$ electrons traveling on helical orbits in a magnetic field, concluding with a $3500 \mathrm{ppt}$ measurement of $g / 2$ [13]. (Here ppt refers to 1 part in $10^{12}$, and ppb refers to 1 part in $10^{9}$.) Research groups at the University of Mainz and the University of Washington (UW) next developed methods to measure the electron magnetic moment using a large number of electrons stored in a Penning trap [14-17]. Out of these efforts came the capability to suspend and detect a single electron in a Penning trap [18] at the UW. A few years later a measurement was made with one electron [19]. Over the next decade these methods were refined, culminating in 


\begin{tabular}{|c|c|c|}
\hline \multicolumn{3}{|c|}{$\mathrm{ppt}=10^{-12}$} \\
\hline 0 & 5 & 10 \\
\hline \multirow{2}{*}{$\stackrel{1-1}{\longmapsto}$} & Harvard (2008) & \\
\hline & Harvard (2006) & UW (1987) \\
\hline \multirow{3}{*}{180} & & \\
\hline & 185 & 190 \\
\hline & $(g / 2-1.00$ & $/ 10^{-12}$ \\
\hline
\end{tabular}

FIG. 1. Measurements $[1,2,4]$ of the dimensionless magnetic moment of the electron, $g / 2$, which is the electron magnetic moment in Bohr magnetons.

the celebrated 1987 measurement already mentioned [4] that reported an uncertainty of $4 \mathrm{ppt}$.

\section{The fine structure constant}

The fine structure constant,

$$
\alpha=\frac{e^{2}}{4 \pi \epsilon_{0} \hbar c},
$$

gives the strength of the electromagnetic coupling in the lowenergy limit. The energy scales for atoms are set by powers of $\alpha$ times the electron rest energy, $m c^{2}$. For hydrogen atoms the binding energy scale is $\alpha^{2} m c^{2}$, the fine structure splitting scale is $\alpha^{4} m c^{2}$, and Lamb shift scale is $\alpha^{5} m c^{2}$. The quantum Hall conductance is proportional to the fine structure constant. The fine structure constant is already a crucial ingredient in our system of fundamental constants [20,21] and it will acquire a more prominent role if plans to redefine the SI system of units [22] go forward.

Section VIIB shows how the new measurements of the electron $g / 2$ determine $\alpha$ to be $[2,23,24]$

$$
\alpha^{-1}=137.035999084(51) \quad[0.37 \mathrm{ppb}] \text {. }
$$

The uncertainty in $\alpha$ is now limited a bit more by the need for a higher-order QED calculation (underway [25]) than by the measurement uncertainty in $g / 2$. The total $0.37 \mathrm{ppb}$ uncertainty in $\alpha$ is now about 12 times smaller than that of the next-most-precise independent method (Fig. 2).

\section{ONE-ELECTRON QUANTUM CYCLOTRON}

\section{A. Electron in a magnetic field}

For an electron in a magnetic field, $g / 2$ is specified by its spin and cyclotron frequencies, $v_{s}$ and $v_{c}$,

$$
\frac{g}{2}=\frac{v_{s}}{v_{c}}=1+\frac{v_{s}-v_{c}}{v_{c}}=1+\frac{v_{a}}{v_{c}}
$$

or equivalently by their difference (the anomaly frequency $v_{a} \equiv v_{s}-v_{c}$ ) and $v_{c}$. Because $v_{s}$ and $v_{c}$ differ by only a partper-thousand, measuring $v_{a}$ and $v_{c}$ to a precision of 1 part in $10^{10}$ gives $g / 2$ to 1 part in $10^{13}$.

Although we cannot measure accurately with one electron in free space because the electron would not stay in one place long enough, two features of determining $g / 2$ are already apparent in Eq. (5). First, one can determine $g / 2$ by measuring a ratio of frequencies. This is fortunate because there is nothing in physics that can be measured more accurately than a frequency (the art of time keeping being developed being so highly developed) except for a ratio of frequencies. Second, although both of these frequencies depend upon the magnetic field, the field dependence drops out of the ratio. The magnetic field thus needs to be stable only on the time scale on which both frequencies can be measured, and no absolute calibration of the magnetic field is required.

\section{B. Electron in a Penning trap}

An ideal Penning trap confines an electron using a magnetic field $B \hat{\mathbf{z}}$ with an additional electrostatic quadrupole potential $V \sim z^{2}-\rho^{2} / 2$ [29]. This potential confines the electron axially with frequency $\bar{v}_{z}$ and shifts the cyclotron frequency from the free-space value $v_{c}$ to $\bar{v}_{c}$. The latter frequency is also slightly shifted by the unavoidable leading imperfections of a real laboratory trap - a misalignment of the symmetry axis of the electrostatic quadrupole and the magnetic field, and quadratic distortions of the electrostatic potential.

The lowest cyclotron energy levels (with quantum numbers $n=0,1, \ldots$ ) and the spin energy levels (with quantum numbers $m_{s}= \pm 1 / 2$ ) (Fig. 3 ) are given by

$E\left(n, m_{s}\right)=\frac{g}{2} h v_{c} m_{s}+\left(n+\frac{1}{2}\right) h \bar{\nu}_{c}-\frac{1}{2} h \delta\left(n+\frac{1}{2}+m_{s}\right)^{2}$.

The third term in Eq. (6) is the leading relativistic correction [29] to the energy levels. Special relativity makes the transition frequency between two cyclotron levels $\left|n, m_{s}\right\rangle \leftrightarrow\left|n+1, m_{s}\right\rangle$ decrease from $\bar{v}_{c}$ to $\bar{v}_{c}+\Delta \bar{v}_{c}$, with the shift

$$
\Delta \bar{\nu}_{c}=-\delta\left(n+1+m_{s}\right)
$$

depending upon the spin state and cyclotron state. This very small shift, with

$$
\delta / v_{c} \equiv h v_{c} /\left(m c^{2}\right) \approx 10^{-9}
$$

is nonetheless significant at our precision. However, an essentially exact treatment of the relativistic shift is possible because single quantum transitions are resolved. The relativistic shift thus contributes no uncertainty to our measurement. This is a key advantage of the quantum cyclotron over previous measurements [4], in which an unknown distribution of cyclotron states was excited [30], each with a different relativistic shift.

To determine $g / 2$, we must rewrite Eq. (5) in terms of measurable frequencies of an electron bound in the trap. The needed free-space cyclotron frequency, $v_{c}=e B /(2 \pi m)$, is

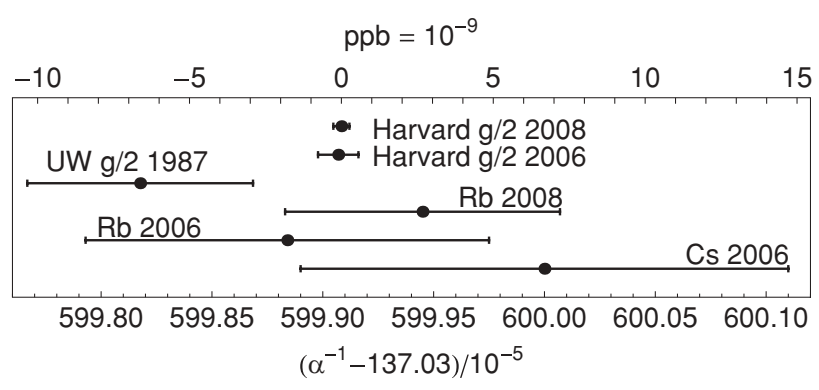

FIG. 2. The most precise $\alpha$ determinations [1,2,26-28]. 


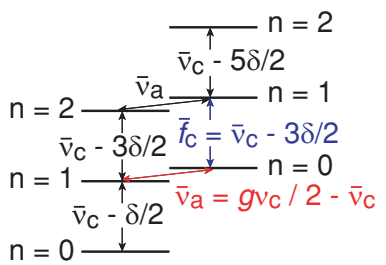

$$
\begin{aligned}
& m_{\mathrm{s}}=-1 / 2 \quad m_{\mathrm{s}}=1 / 2
\end{aligned}
$$

FIG. 3. (Color online) Lowest cyclotron and spin levels of an electron in a Penning trap.

deduced by use of the Brown-Gabrielse invariance theorem [31],

$$
\left(v_{c}\right)^{2}=\left(\bar{v}_{c}\right)^{2}+\left(\bar{v}_{z}\right)^{2}+\left(\bar{v}_{m}\right)^{2} .
$$

The three measurable eigenfrequencies on the right include the cyclotron frequency $\bar{v}_{c}$ for the quantum cyclotron motion we have been discussing. The second measurable eigenfrequency is the axial oscillation frequency $\bar{v}_{z}$ for the nearly harmonic, classical electron motion along the direction of the magnetic field. The third measurable eigenfrequency is the magnetron oscillation frequency for the classical magnetron motion along the circular orbit for which the electric field from the trap electrodes cancels the motional electric field (so the total radial Lorentz force vanishes).

The invariance theorem applies for a perfect Penning trap, but also in the presence of the unavoidable imperfection shifts of the eigenfrequencies for a real trap. This theorem, together with the well-defined hierarchy of trap eigenfrequencies, $\bar{v}_{c} \gg \bar{v}_{z} \gg \bar{v}_{m} \gg \delta$, yields an approximate expression that is sufficient at our accuracy. We thus determine the electron $g / 2$ using

$$
\frac{g}{2}=\frac{\bar{v}_{c}+\bar{v}_{a}}{v_{c}} \simeq 1+\frac{\bar{v}_{a}-\bar{v}_{z}^{2} /\left(2 \bar{f}_{c}\right)}{\bar{f}_{c}+3 \delta / 2+\bar{v}_{z}^{2} /\left(2 \bar{f}_{c}\right)}+\frac{\Delta g_{\text {cav }}}{2} .
$$

The two frequencies that must be measured to the highest precision are the cyclotron and anomaly transition frequencies,

$$
\begin{gathered}
\bar{f}_{c} \equiv \bar{v}_{c}-\frac{3}{2} \delta, \\
\bar{v}_{a} \equiv \frac{g}{2} v_{c}-\bar{v}_{c},
\end{gathered}
$$

shown in Fig. 3. A lower precision measurement of the axial frequency $\bar{v}_{z}$ is also required, as is a cavity shift $\Delta g_{\text {cav }} / 2$ (from the interaction of the cyclotron motion and the trap cavity, discussed in Sec. V).

\section{EXPERIMENTAL REALIZATION}

\section{A. Cylindrical Penning trap}

A cylindrical Penning trap (Fig. 4) is the key device that makes these measurements possible. It was invented [6] and demonstrated [7] to provide boundary conditions that produce a controllable and understandable radiation field within the trap cavity. Spontaneous emission can be significantly inhibited at the same time as corresponding shifts of the electron's oscillation frequencies are avoided. The latter has not been possible [32] with the hyperbolic Penning traps of earlier

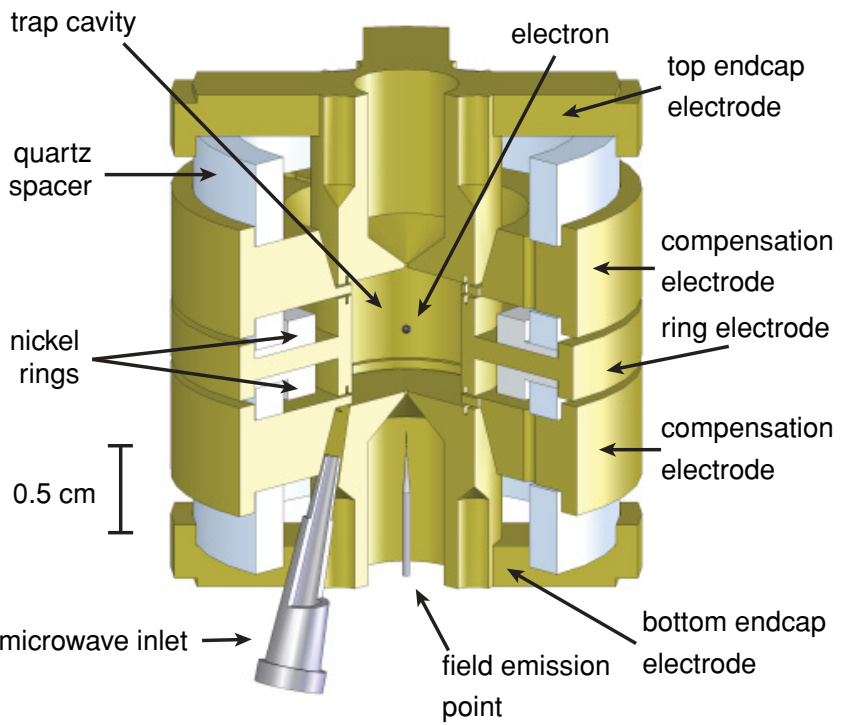

FIG. 4. (Color) Section view of the cylindrical Penning trap cavity used to confine a single electron and inhibit spontaneous emission. The electrodes facing the electron are cylindrically symmetric except for the compensation electrodes, each of which are split vertically in half. Microwaves with a $2 \mathrm{~mm}$ wavelength enter the trap through the squeezed tube that is the microwave inlet.

experiments [4], which have electrodes approximating the equipotentials of an electrostatic quadrupole.

The first function of the trap electrodes is to produce a very good approximation to an electrostatic quadrupole potential. This requires careful choice of the relative geometry of the electrodes [6]. The electrodes of the cylindrical trap are symmetric under rotations about the center axis $(\hat{\mathbf{z}})$, which is parallel to the spatially uniform magnetic field $(B \hat{\mathbf{z}})$. The potential applied between the endcap electrodes and the ring electrode provides the basic trapping potential and sets the axial frequency $\bar{v}_{z}$ of the nearly harmonic oscillation of the electron parallel to the magnetic field. (See Table I for typical parameters and frequencies.) The potential applied to the compensation electrodes is adjusted to tune the shape of the potential, to make the oscillation as harmonic as possible. The tuning does not change $\bar{v}_{z}$ very much owing to an orthogonalization $[6,33]$ that arises from the geometry choice. What we found was that one electron could be observed within a cylindrical Penning trap with as good or better signal-to-noise ratio than was realized in hyperbolic Penning traps.

\section{B. Radiation field in a cylindrical trap}

The second function of the trap electrodes is to form a microwave cavity whose radiation properties are well understood and controlled. The resonant radiation modes of a perfect right circular cylinder are the familiar transverse electric (TE) and transverse magnetic (TM) radiation modes for such a geometry. In the real trap cavity, the perturbation caused by the small space between the electrodes is minimized by the use of "choke flanges"- small channels that tend to reflect the radiation leaking out of the trap back to cancel itself, and thus to minimize the losses from the trap. The measured radiation modes (Fig. 10) are close enough to the calculated frequencies for a perfect cylindrical cavity that we have been 
able to identify more than 100 different radiation modes for such trap cavities [8]. The spatial properties of the electric and magnetic field for the radiation that builds up within the cavity are thus quite well understood. Some of the modes couple to cyclotron motion of an electron centered in the cavity, others couple to the spin of a centered electron, and still others have the symmetry that we hope will one day allow us to sideband cool the axial motion [12].

A right circular cylinder of diameter $2 \rho_{0}$ and height $2 z_{0}$ admits two classes of electromagnetic fields, transverse electric $\mathrm{TE}_{m n p}$ and transverse magnetic $\mathrm{TM}_{m n p}$, each identified by three indices that describe their specific geometry (see, e.g., [34], Sec.8.7). These TE and TM modes have characteristic frequencies,

$$
\begin{aligned}
\mathrm{TE}: \omega_{m n p} & =c \sqrt{\left(\frac{x_{m n}^{\prime}}{\rho_{0}}\right)^{2}+\left(\frac{p \pi}{2 z_{0}}\right)^{2}} \\
\mathrm{TM}: \omega_{m n p} & =c \sqrt{\left(\frac{x_{m n}}{\rho_{0}}\right)^{2}+\left(\frac{p \pi}{2 z_{0}}\right)^{2}},
\end{aligned}
$$

that are indexed with integers

$$
\begin{gathered}
m=0,1,2, \ldots, \\
n=1,2, \ldots, \\
p=\mathrm{TE}: 1,2, \ldots ; \quad \mathrm{TM}: 0,1, \ldots,
\end{gathered}
$$

and are functions of the $n$th zeros of Bessel functions and their derivatives

$$
\begin{aligned}
& J_{m}\left(x_{m n}\right)=0, \\
& J_{m}^{\prime}\left(x_{m n}^{\prime}\right)=0 .
\end{aligned}
$$

The zeros force the boundary conditions at the cylindrical wall. All but the $m=0$ modes are doubly degenerate.

Of primary concern is the magnitude of the transverse electric fields since only these components couple to cyclotron motion. For both TE and TM modes, the transverse components of $\mathbf{E}$ are proportional to

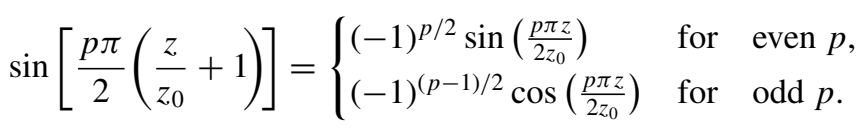

For an electron close to the cavity center, $(z \approx 0)$, only modes with odd $p$ thus have any appreciable coupling.

The transverse components of the electric fields are also proportional to either the order- $m$ Bessel function times $m / \rho$ or to the derivative of the order- $m$ Bessel function. Close to the cavity center $(\rho \approx 0)$,

$$
\begin{gathered}
\frac{m}{\rho} J_{m}\left(x_{m n}^{(\prime)} \frac{\rho}{\rho_{0}}\right) \sim\left\{\begin{array}{lll}
\frac{\rho^{m-1}}{(m-1) !}\left(\frac{x_{m n}^{(\prime)}}{2 \rho_{0}}\right)^{m} & \text { for } & m>0 \\
0 & \text { for } & m=0
\end{array},\right. \\
\frac{x_{m n}^{(\prime)}}{\rho_{0}} J_{m}^{\prime}\left(x_{m n}^{(\prime)} \frac{\rho}{\rho_{0}}\right) \sim\left\{\begin{array}{lll}
\frac{\rho^{m-1}}{(m-1) !}\left(\frac{x_{m n}^{(\prime)}}{2 \rho_{0}}\right)^{m} & \text { for } & m>0 \\
-\frac{x_{0 n}^{(\prime)}}{2 \rho_{0}^{2}} \rho & \text { for } & m=0
\end{array} .\right.
\end{gathered}
$$

In the limit $\rho \rightarrow 0$, all but the $m=1$ modes vanish.
For a perfect cylindrical cavity the only radiation modes that couple to an electron perfectly centered in the cavity are $\mathrm{TE}_{1 n \text { (odd) }}$ and $\mathrm{TM}_{1 \text { (odd) }}$. If the electron is moved slightly off center axially it will begin to couple to radiation modes with $m n p=1 n$ (even). If the electron is moved slightly off-center radially it similarly begins to couple to modes with $m \neq 1$.

\section{C. $100 \mathrm{mK}$ and $5 \mathrm{~T}$}

The trap cavity is cooled to $0.1 \mathrm{~K}$ or below via thermal contact with the mixing chamber of an Oxford Instruments Kelvinox 300 dilution refrigerator (Fig. 5). They are housed within a separate vacuum enclosure that is entirely at the base temperature. Measurements on an apparatus with a similar design but at $4.2 \mathrm{~K}$ found the vacuum in the enclosure to be better than $5 \times 10^{-17}$ torr [35]. Our much lower temperature should make our background gas pressure much lower. We are able to keep one electron suspended in our apparatus for as long as desired-regularly months at a time. Substantial reservoirs for liquid helium and liquid nitrogen make it possible to keep the trap cold for five to seven days before the disruption of adding more cryogens is required.

The trap electrodes and their vacuum container are located within a superconducting solenoid (Fig. 5) that makes a very homogeneous magnetic field over the interior volume of the trap cavity. A large dewar sitting on top of the solenoid dewar provides the helium needed around the dilution refrigerator below. The superconducting solenoid is entirely self-contained, with a bore that can operate from room temperature down to $77 \mathrm{~K}$. It possesses shim coils capable of creating a field homogeneity better than a part in $10^{8}$ over a $1 \mathrm{~cm}$ diameter sphere and has a passive "shield" coil that reduces fluctuations in the ambient magnetic field [36,37]. When properly energized (and after the steps described in the next section have been taken) it achieves field stability better than a part in $10^{9}$ per hour. We regularly observe drifts below $10^{-9}$ per night.

\section{Attaining high stability}

Measuring the electron $g / 2$ with a precision of parts in $10^{13}$ requires careful attention to making a stable trapping potential. Even more important is a stable magnetic field since the frequencies $\bar{f}_{c}$ and $\bar{v}_{a}$ that we measure are both proportional to $B$, and we are not able to measure these frequencies at exactly the same time.

A major defense against external field fluctuations is a high magnetic field. This makes fluctuations from outside sources relatively smaller. The largest source of ambient magnetic noise is a subway that produces $50 \mathrm{nT}(0.5 \mathrm{mG}, 10 \mathrm{ppb})$ fluctuations in our laboratory and that would limit us to four hours of data taking per day (when the subway stops running) if we did not shield the electron from them. Eddy currents in the high-conductivity aluminum and copper cylinders of the Dewars and the magnet bore shield high-frequency fluctuations [38]. For slower fluctuations, the aforementioned shelf-shielding solenoid [36] has the correct geometry to make the central field always equal to the average field over the solenoid cross-section. This translates flux conservation into 


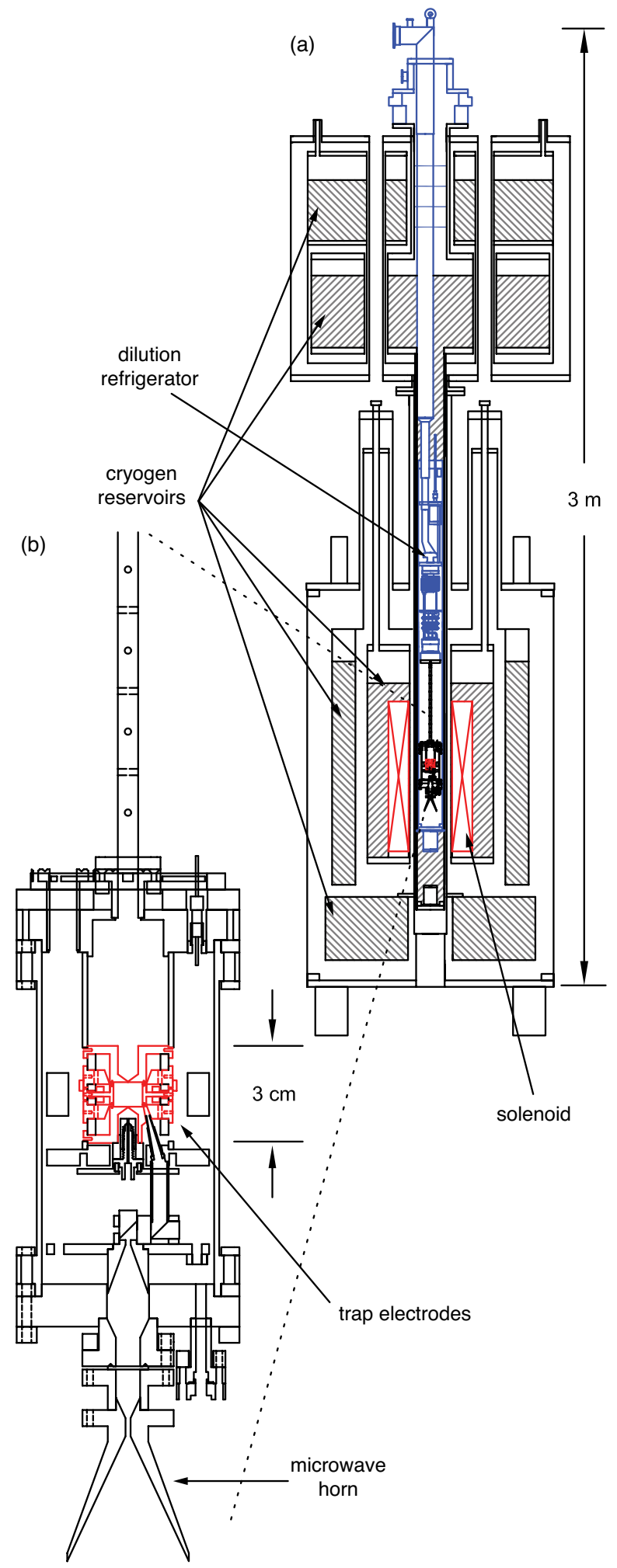

FIG. 5. (Color) An overview of the apparatus (a), including a 10-times enlarged view (b) of the vacuum enclosure for the Penning trap electrodes. The solenoid and electrodes are in red. The dilution refrigerator is in blue. Cryogen spaces are hatched.

central-field conservation, shielding external fluctuations by more than a factor of 150 [37].

Stabilizing the field produced by the solenoid requires that care is taken when the field value is changed, since changing
TABLE I. Typical trap parameters as well as frequencies and damping rates for each degree of freedom. The damping rates include coupling to a detection circuit for $\gamma_{z}$ and inhibited spontaneous emission for $\gamma_{c}$. To sample the radiation modes of the electrode cavity, we change $B$, and hence $\bar{v}_{c}, \gamma_{c}$, and $v_{s}$ (see Sec. V).

\begin{tabular}{lcccc}
\hline \hline magnetic field & $B$ & $5.36 \mathrm{~T}$ & & \\
electrode potential & $V_{0}$ & $101.4 \mathrm{~V}$ & & \\
electrode cavity radius & $\rho_{0}$ & $4.5 \mathrm{~mm}$ & & \\
electrode cavity height & $2 z_{0}$ & $7.7 \mathrm{~mm}$ & & \\
\hline magnetron & $\bar{v}_{m}$ & $133 \mathrm{kHz}$ & $\gamma_{m}^{-1}$ & $4 \mathrm{Gyr}$ \\
axial & $\bar{v}_{z}$ & $200 \mathrm{MHz}$ & $\gamma_{z}^{-1}$ & $0.2 \mathrm{~s}$ \\
cyclotron & $\bar{v}_{c}$ & $150.0 \mathrm{GHz}$ & $\gamma_{c}^{-1}$ & $3.7 \mathrm{~s}$ \\
spin & $v_{s}$ & $150.2 \mathrm{GHz}$ & $\gamma_{s}^{-1}$ & $2 \mathrm{yr}$ \\
\hline \hline
\end{tabular}

the current in the solenoid alters the forces between windings. Resulting stresses can take months to stabilize if the coil is not prestressed by "over-currenting" the magnet. Our recipe is to overshoot the target value by a few percent of the change, undershoot by a similar amount, and then move to the desired field, pausing several minutes after each change.

The apparatus in Fig. 5 evolved historically rather than being designed for maximum magnetic field stability in the final configuration. Because the solenoid and the trap electrodes are suspended from widely separated support points, temperature and pressure changes can cause the electrodes to move relative to the solenoid. Apparatus vibrations can do the same. Insofar as the magnetic field is not perfectly homogeneous, despite careful adjusting of the persistent currents in ten superconducting shim coils, such relative motion changes the field seen by the electron.

To counteract this, we regulate the five $\mathrm{He}$ and $\mathrm{N}_{2}$ pressures in the cryostats to $\approx 50 \mathrm{ppm}$ to maintain the temperature of both the bath and the solenoid itself $[39,40]$. Recently we also relocated the dilution refrigerator vacuum pumps to an isolated room at the end of a $12 \mathrm{~m}$ pipe run. This reduced vibration by more than an order of magnitude at frequencies related to the pump motion and reduced the noise level for the experimenters but did not obviously improve the $g / 2$ data.

Because some of the structure establishing the relative location of the trap electrodes and the solenoid is at room temperature, changes in room temperature can move the electron in the magnetic field. The laboratory temperature routinely cycles $1-2 \mathrm{~K}$ daily, so we house the apparatus in a large, insulated enclosure within which we actively regulate the air temperature to $0.1 \mathrm{~K}$. A refrigerated circulating bath (ThermoNeslab RTE-17) pumps water into the regulated zone and through an automobile transmission fluid radiator, heating and cooling the water to maintain constant air temperature. Fans couple the water and air temperatures and keep a uniform air temperature throughout.

The choice of materials for the trap electrodes and its vacuum container is also crucial to attaining high field stability [1,41]. Copper trap electrodes, for example, have a nuclear paramagnetism at $0.1 \mathrm{~K}$ that makes the electron see a magnetic field that changes at an unacceptable level with very small changes in trap temperature. We thus use only low-Curie-constant materials such as silver, quartz, titanium, 
and molybdenum at the refrigerator base temperature and we regulate the mixing chamber temperature to better than $1 \mathrm{mK}$.

A stable axial frequency is also extremely important since small changes in the measured axial frequency are the detected consequences of the one-quantum transitions of the cyclotron and spin energy (as will be discussed in Sec. III F). A trapping potential without thermal fluctuations is provided by a charged capacitor $(10 \mu \mathrm{F})$. Fluctuations in its very low leakage current, in its capacitance, and in the mechanical strain across the capacitor are minimized by thermally coupling the capacitor to the well-regulated low temperature of the dilution refrigerator. We add to or subtract from the charge on the capacitor using $50 \mathrm{~ms}$ current pulses sent to the capacitor through a $100 \mathrm{M} \Omega$ resistor as needed to keep the measured axial frequency constant. Because of the orthogonalized trap design [6] already discussed, the potential applied to the compensation electrodes (to make the electron see as close to a pure electrostatic quadrupole potential as possible) has little effect upon the axial frequency.

\section{E. One electron: Its motions and damping}

We load a single electron using an electron beam from an atomically sharp tungsten field-emission tip. A hole in the bottom endcap electrode admits the beam, which hits the top endcap electrode and releases gas atoms cryopumped on the surface. Collisions between the beam and gas atoms allows an electron to fall into the trap. Adjusting the beam energy and the time it is left on controls the number of electrons loaded.

The electron has three motions in the Penning trap formed by the $B=5.4 \mathrm{~T}$ magnetic field, and the electrostatic quadrupole potential. The cyclotron motion in the trap has a frequency $\bar{v}_{c} \approx 150 \mathrm{GHz}$. The axial frequency, for the harmonic oscillator parallel to the magnetic field direction, is $\bar{v}_{z} \approx 200 \mathrm{MHz}$. A circular magnetron motion, perpendicular to $\mathbf{B}$, has an oscillation frequency, $\bar{v}_{m} \approx 133 \mathrm{kHz}$. The spin precession frequency, which we do not measure directly, is a part-per-thousand higher than the cyclotron frequency. The frequency difference is the anomaly frequency, $\bar{v}_{a} \approx$ $174 \mathrm{MHz}$, which we do measure directly.

The undamped spin motion is essentially uncoupled from its environment [29]. The cyclotron motion is only weakly damped. By controlling the cyclotron frequency relative to that of the cavity radiation modes, we alter the density of radiation states and inhibit the spontaneous emission of synchrotron radiation $[29,42]$ by 10 to 50 times the $(90 \mathrm{~ms})^{-1}$ free-space rate. Blackbody photons that could excite the electron from the cyclotron ground state are eliminated because the trap cavity is cooled by the dilution refrigerator to $100 \mathrm{mK}$ [3]. The axial motion is cooled by a resonant circuit at a rate $\gamma_{z} \approx(0.2 \mathrm{~s})^{-1}$ to as low as $230 \mathrm{mK}$ (from $5 \mathrm{~K}$ ) when the detection amplifier is off. The magnetron radius is minimized with axial sideband cooling [29].

\section{F. QND detection}

Quantum nondemolition (QND) detection has the property that repeated measurements of the energy of the system will not change the quantum state of the system [43]. This is crucial for our detection of one-quantum transitions in the cyclotron motion, where we do not want the detection system to produce the transitions that we observe. In this section we discuss the QND coupling and in the next section our readout system.

Detecting a single $150 \mathrm{GHz}$ photon from the decay of one cyclotron energy level to the level below is very difficult. One photon detectors are not readily available at this high microwave frequency, and it would be difficult to cover the solid angle into which the photon could be emitted. Instead we get the single-photon sensitivity by coupling the cyclotron motion to the orthogonal axial motion at $200 \mathrm{MHz}$, a frequency at which we are able to make sensitive detection electronics [44]. The QND nature of the detection means the thermally driven axial motion of the electron does not change the state of the cyclotron motion.

We use a magnetic bottle gradient that is familiar from plasma physics and from earlier electron measurements $[4,45]$,

$$
\Delta \mathbf{B}=B_{2}\left[\left(z^{2}-\rho^{2} / 2\right) \hat{\mathbf{z}}-z \rho \hat{\boldsymbol{\rho}}\right],
$$

with $B_{2}=1540 \mathrm{~T} / \mathrm{m}^{2}$. The gradient arises from a pair of thin nickel rings (Fig. 4) that are completely saturated in the strong field from the superconducting solenoid. To lowest order the rings modify $B$ by $\approx-0.7 \%$ - merely changing the magnetic field that the electron experiences without affecting our measurement.

The formal requirement for a QND measurement is that the Hamiltonian of the quantum system (i.e., the cyclotron Hamiltonian) and the Hamiltonian describing the interaction of the quantum system and the classical measurement system must commute. The Hamiltonian that couples the quantum cyclotron and spin motions to the axial motion does so. It has the form $-\mu B$, where $\mu$ is the magnetic moment associated with the cyclotron motion or the spin. The coupling Hamiltonian thus has a term that goes as $\mu z^{2}$. This term has the same spatial symmetry as does the axial Hamiltonian, $H=\frac{1}{2} m\left(2 \pi \bar{v}_{z}\right)^{2} z^{2}$. A change in the magnetic moment that takes place from a one-quantum change in the cyclotron or spin magnetic moment thus changes the observed axial frequency of the suspended electron.

The result is that the frequency of the axial motion $\bar{v}_{z}$ shifts by

$$
\Delta \bar{v}_{z}=\delta_{B}\left(n+m_{s}\right),
$$

in proportion to the cyclotron quantum number $n$ and the spin quantum number $m_{s}$. Figure 6 shows the $\Delta \bar{\nu}_{z}=4 \mathrm{~Hz}$ shift in the $200 \mathrm{MHz}$ axial frequency that takes place for one-quantum changes in cyclotron Fig. 6(a) and spin energy Fig. 6(b). The $20 \mathrm{ppb}$ shift is typically observed with a $0.25 \mathrm{~s}$ averaging time.

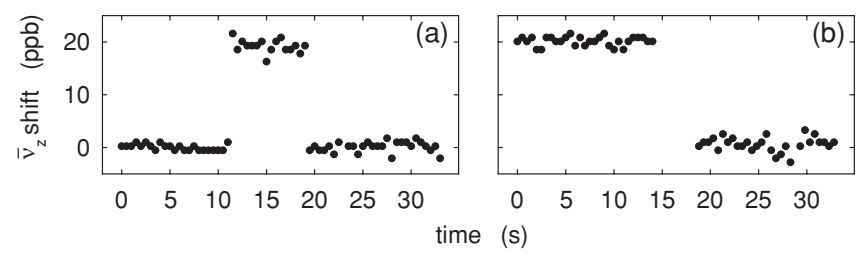

FIG. 6. A one-quantum cyclotron transition (a) and a spin flip (b) observed with a QND measurement through shifts in the axial frequency. 


\section{G. One-electron self-excited oscillator}

Cyclotron excitations and spin flips are generally induced while the detection system is off, as will be discussed. After an attempt to excite the cyclotron motion or to flip the spin has been made, the detection system is then turned on to detect the state of the system. Spontaneous emission of synchrotron radiation from the cyclotron motion is inhibited (Sec. III H) to give the time that is needed. The small axial frequency shifts which signal changes in cyclotron quantum number or spin are measured at the required precision using a one-electron self-excited oscillator [11] that turns on rapidly.

The $200 \mathrm{MHz}$ axial frequency lies in the radio-frequency (rf) range which is more experimentally accessible than the microwave range of the $150 \mathrm{GHz}$ cyclotron and spin frequencies, as mentioned. Nevertheless, standard rf techniques must be carefully tailored for our low-noise, cryogenic experiment. The electron axial oscillation induces image currents in the trap electrodes that are proportional to the axial velocity of the electron $[29,46]$. An inductor is placed in parallel with the capacitance between two trap electrodes to cancel the reactance of the capacitor which would otherwise short out the induced signal. The rf loss in the tuned circuit that is formed is an effective resistance that damps the axial motion.

The voltage that the electron motion induces across this effective resistance is amplified with two cryogenic amplifiers [44]. The heart of each amplifier is a single-gate high electron mobility transistor (Fujitsu FHX13LG).

The first amplifier is at the $100 \mathrm{mK}$ dilution refrigerator base temperature. Operating this amplifier without crashing the dilution refrigerator requires operating with a power dissipation in the field-effect transistor (FET) that is three orders of magnitude below the transistor's $10 \mathrm{~mW}$ design dissipation. The effective axial temperature for the electron while current is flowing through the FET is about 5 $\mathrm{K}$, well above the ambient temperature. Very careful heat sinking reduces the temperature of the circuit when the current is stopped, cooling the effective axial temperature of the electron below $350 \mathrm{mK}$ in approximately $1 \mathrm{~s}$. Cyclotron excitations and spin flips are induced only when the axial motion is so cooled, since the electron is then making the smallest possible excursion in the magnetic field gradient.

The second amplifier is mounted on the nominally $600 \mathrm{mK}$ still of the dilution refrigerator. This amplifier counteracts the attenuation of thermally isolating but lossy stainless steel transmission line that carries the amplified signal out of the refrigerator. The second amplifier boosts the signal above the noise floor of the following room-temperature amplifier.

We feed this signal back to trap electrodes as a drive. Because the induced signal is proportional to the electron's axial velocity, this feedback alters the axial damping force, a force that is also proportional to the electron velocity. Changing the feedback gain thus changes the damping rate. As the gain increases, the damping rate decreases if the feedback phase is appropriately adjusted, as does the effective axial temperature of the electron, in accord with the fluctuation dissipation theorem [47]. The invariant ratio of the separately measured damping rate and the effective temperature has been demonstrated [48], thereby also demonstrating that the amplifier adds very little noise to the feedback.

Setting the feedback gain to make the drive exactly cancel the damping in the attached circuit could sustain a large axial oscillation amplitude, in principle. However, the gain cannot be perfectly adjusted and noise fluctuations will always drive the axial oscillation exponentially away from equilibrium. We thus stabilize the oscillation amplitude using a digital signal processor (DSP) that Fourier transforms the signal, and adjusts the feedback gain in real time to keep the signal at a fixed value. The corresponding oscillation amplitude was selected to minimize the dependence of the oscillation frequency upon the oscillation amplitude [11].

This one-particle self-excited oscillator is turned on after an attempt has been made to increase the cyclotron energy by one quantum, or to flip the spin. The frequency of the axial oscillation that rapidly stabilizes at a large and easily detected amplitude is then measured. Small shifts in this frequency reveal whether the cyclotron motion has been excited or whether the spin has flipped, as illustrated in Fig. 6.

\section{H. Inhibited spontaneous emission}

One of the early papers in what has come to be known as cavity electrodynamics was an observation of inhibited spontaneous emission within a Penning trap [42] - the first time that inhibited spontaneous emission was observed within a cavity and with only one particle-as anticipated earlier $[49,50]$. As already mentioned, the cylindrical Penning trap [6] was invented to provide boundary conditions that would allow the control of the electron-cavity coupling, using an understandable geometry that allows the calculation of cavity shifts to the electron's cyclotron frequency.

The spontaneous emission rate can be easily measured directly, by making a histogram of the time the electron spends in the first excited state after being excited by a microwave drive injected into the trap cavity. Figure 7 shows a sample histogram which fits well to an exponential (solid curve) with a lifetime of $3.4 \mathrm{~s}$ in this example.

Stimulated emission is avoided by making these observations only when the cavity is at low temperature so that effectively no black body photons are present. The detector

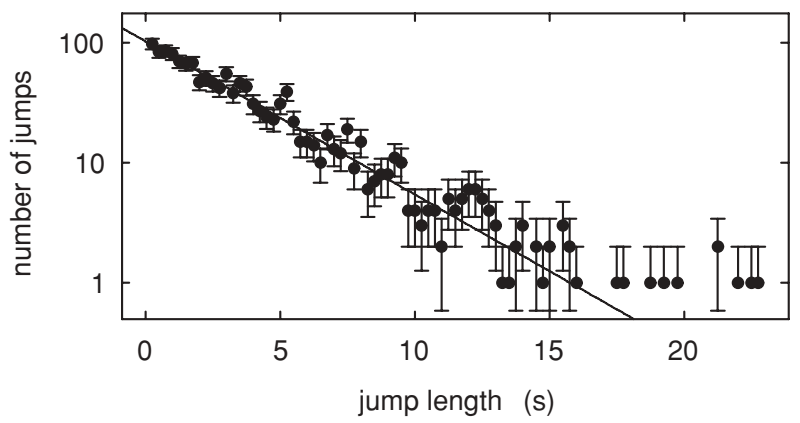

FIG. 7. Histogram of the time that the electron spends in the first excited state with an exponential fit. The decay time, 3.4(1) s in this example, depends on how close the cyclotron frequency is to neighboring radiation modes of the trap cavity. Lifetimes as long as $16 \mathrm{~s}$ have been observed. 
makes thermal fluctuations of the axial oscillation amplitude, and these in turn make the cyclotron frequency fluctuate. For measuring the cyclotron decay time, however, this does not matter because the fluctuations in axial amplitude are small compared to the $2 \mathrm{~mm}$ wavelength of the radiation that excites the cyclotron motion.

The spontaneous emission rate into free space is [29]

$$
\gamma_{c 0}=\frac{1}{4 \pi \epsilon_{0}} \frac{4 e^{2}}{3 m c^{3}} \frac{\bar{\omega}_{c}^{3}}{\bar{\omega}_{c}-\bar{\omega}_{m}} \approx \frac{1}{89 \mathrm{~ms}} .
$$

The measured rate in the example of Fig. 7 is thus suppressed by a factor of 38 . The density of states within a finite cylindrical trap cavity is not that of free space. The continuous free-space density of states is generally calculated by considering the limit of a cavity whose boundary conditions are extended to infinity. For a finite cavity the density of states for the radiation remains peaked at the resonance frequencies of the resonant, electromagnetic radiation modes of the cavity, and falls to very low values between the modes. We attain the inhibited spontaneous emission by tuning the magnetic field so that the cyclotron frequency is far from radiation modes. (Adjusting the magnetic field in a way that most rapidly restores field stability is discussed earlier.) With the right choice of magnetic field we have increased the lifetime to $16 \mathrm{~s}$, which is a cavity suppression of spontaneous emission by a factor of 180 .

In Sec. V D we report on using the direct measurements of the radiation rate for electron cyclotron motion to probe the radiation modes of the cavity, with the radiation rate increasing sharply at frequencies that approach a resonant mode of the cavity.

\section{Quantum jump spectroscopy}

We probe the cyclotron and anomaly resonances with quantum jump spectroscopy, in which we apply a drive in discrete frequency steps, checking between applications for a one-quantum transition and building a histogram of the ratio of excitations to attempts at each frequency. Figure 8 shows the observed lineshapes upon which our best measurement is based. We discuss how the measured points are obtained first, and then discuss theoretical lineshapes in Sec. IV.

A typical data run consists of alternating scans of the cyclotron and anomaly lines and occurs at night, with daytime runs only possible on Sundays and holidays when the ambient magnetic field noise is lower. Interleaved every three hours among these scans are periods of magnetic field monitoring to track long-term drifts using the electron itself as the magnetometer. In addition, we continuously monitor over fifty environmental parameters such as refrigerator temperatures, cryogen pressures and flows, and the ambient magnetic field in the laboratory so that we may screen data for abnormal conditions and troubleshoot problems.

Cyclotron transitions are driven by injecting microwaves into the cavity. The microwaves originate as a $15 \mathrm{GHz}$ drive from a signal generator (Agilent E8251A) whose low-phasenoise, $10 \mathrm{MHz}$ oven-controlled crystal oscillator serves as the time base for all frequencies in the experiment. After passing through a waveguide that removes all subharmonics, the signal enters a microwave circuit that includes an impact ionization avalanche transit-time (IMPATT) diode, which multiplies the

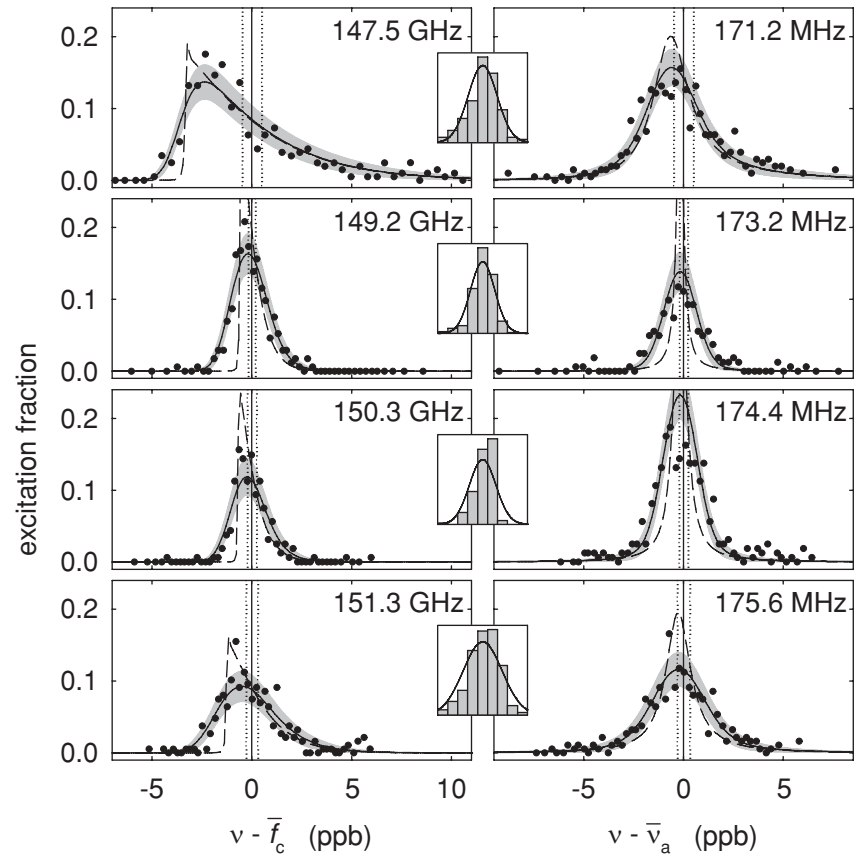

FIG. 8. Quantum-jump spectroscopy lineshapes for cyclotron (left) and anomaly (right) transitions with maximum-likelihood fits to broadened lineshape models (solid) and inset resolution functions (solid) and edge-tracking data (histogram). Vertical lines show the 1- $\sigma$ uncertainties for extracted resonance frequencies. Corresponding unbroadened lineshapes are dashed. Gray bands indicate 1- $\sigma$ confidence limits for distributions about broadened fits. All plots share the same relative frequency scale.

frequency by ten and outputs the $\bar{f}_{c}$ drive at a power of $2 \mathrm{~mW}$. Voltage-controlled attenuators provide control of the strength of the drive. The microwaves from a room temperature horn travel through a series of several teflon lenses, each about ten wavelengths in diameter. The microwaves enter a horn at 100 $\mathrm{mK}$ (Fig. 5) and travel though a cylindrical wave guide that is gradually deformed to be a thin oval to better match the cross sectional area of a gap between two trap electrodes (Fig. 4).

Anomaly transitions are driven by a potential oscillating near $\bar{v}_{a}$ that is applied between the endcap and ring electrodes to drive off-resonant axial motion through the magnetic bottle gradient [Eq. (19)]. The gradient's $z \rho \hat{\boldsymbol{\rho}}$ term mixes the driven oscillation of $z$ at $\bar{v}_{a}$ with that of $\rho$ at $\bar{f}_{c}$ to produce an oscillating magnetic field perpendicular to $\mathbf{B}$ as needed to flip the spin. The axial amplitude required to produce the desired transition probability is too small to affect the lineshape (Sec. VI); nevertheless, we apply a detuned drive of the same strength during cyclotron attempts so the electron samples the same magnetic gradient.

Quantum jump spectroscopy of each resonance follows the same procedure. With the electron prepared in the spin-up ground state $\left|0, \frac{1}{2}\right\rangle$, the magnetron radius is reduced with $1.5 \mathrm{~s}$ of strong sideband cooling at $\bar{v}_{z}+\bar{v}_{m}$ with the self-excited oscillator (SEO) turned off immediately and the detection amplifiers turned off after $0.5 \mathrm{~s}$. After an additional $1 \mathrm{~s}$ to allow the axial motion to thermalize with the tuned circuit, we apply a $2 \mathrm{~s}$ pulse of either a cyclotron drive near $\bar{f}_{c}$ or an anomaly drive near $\bar{v}_{a}$ with the other drive applied simultaneously but detuned far from resonance. The detection 
electronics and SEO are turned back on; after waiting $1 \mathrm{~s}$ to build a steady-state axial amplitude, we measure $\bar{v}_{z}$ and look for a $20 \mathrm{ppb}$ shift up (from a cyclotron transition) or down (from an anomaly transition followed by a spontaneous decay to $\left.\left|0,-\frac{1}{2}\right\rangle\right)$ in frequency. The inhibition of spontaneous emission by the cavity provides the time needed to observe cyclotron transitions before decay. The several-cyclotron lifetimes wait for a spontaneous decay after an anomaly attempt is the rate-limiting step in the spectroscopy. After a successful anomaly transition and decay, simultaneous cyclotron and anomaly drives pump the electron back to $\left|0, \frac{1}{2}\right\rangle$. All timing is done in hardware with a pulse generator. We probe each resonance line with discrete excitation attempts spaced in frequency by approximately $10 \%$ of the linewidth. We step through each drive frequency on the $\bar{f}_{c}$ line, then each on the $\bar{v}_{a}$ line, and repeat.

\section{J. The electron as magnetometer}

Slow drifts of the magnetic field are corrected using the electron itself as a magnetometer. Accounting for these drifts allows the combination of data taken over many days, giving a lineshape signal-to-noise that allows the systematic investigation of lineshape uncertainties at each field. For a half-hour at the beginning and end of a run and again every three hours throughout, we alter our cyclotron spectroscopy routine by applying a stronger drive at a frequency below $\bar{f}_{c}$. Using the same timing as above but a ten-times-finer frequency step, we increase the drive frequency until observing a successful transition. We then jump back 60 steps and begin again. Fitting a polynomial to such cyclotron-edge tracking data allows the normalization of the raw cyclotron and anomaly data to a common magnetic field.

\section{K. Measuring the axial frequency}

Our expression for $g / 2$ [Eq. (10)] requires a measurement of the axial frequency $\bar{v}_{z}$. For a relative uncertainty in $g$ below $0.1 \mathrm{ppt}$, we must know $\bar{v}_{z}$ to better than $50 \mathrm{ppb}$, or $10 \mathrm{~Hz}$. This is easily done.

While we routinely measure $\bar{v}_{z}$ during QND detection of cyclotron and spin states, this self-excited oscillation frequency includes an amplitude-dependent anharmonic shift from the low-amplitude, thermally excited axial motion during cyclotron and anomaly excitation attempts. The shift is typically a few hertz. We account for this shift by directly measuring the thermal axial frequency with the amplifiers on; the axial resonance appears as a narrow dip where the electron has "shorted-out" the amplifier noise [46]. This dip frequency is negligibly different than the slightly lower-amplitude one that pertains during cyclotron and anomaly excitation, which occurs with the amplifiers off. All additional shifts from interaction with the amplifier or anomaly-drive-induced power shifts [51] are negligible at our precision. The uncertainty arising from the measurement of $\bar{v}_{z}$ is not significant.

\section{CYCLOTRON AND ANOMALY FREQUENCIES FROM RESONANCES}

Quantum jump spectroscopy gives a measurement of the number of single-quantum jumps as a function of the frequency of the driving force that makes transitions between two distinct quantum states. From such resonance lineshapes (e.g., Fig. 8) we must deduce some values of the cyclotron and anomaly frequencies that can be used to determine $g / 2$ from Eq. (10). These lineshapes (Sec. IV A) are primarily due to thermal fluctuations of the electron's position within the magnetic bottle gradient. The observed lines are slightly broader than would be expected from thermal fluctuations alone-consistent with a small additional fluctuation in the frequencies (as if there is an additional random fluctuation in the effective magnetic field).

The frequencies that we extract from the observed lineshapes, using a weighted mean method (Sec. IV B), are averages over the values observed in the lineshapes. Insofar as cyclotron and anomaly frequencies are both essentially proportional to the magnetic field, any fluctuations in the effective magnetic field (due to a fluctuating electron position or any other fluctuation in the magnetic field) would average the two frequencies in much the same way. We further argue that the lineshape-weighted mean frequencies are not shifted with respect to each other by the axial dissipative force, quantified by $\gamma_{z}$.

As a extensive consistency check, we also determine $g / 2$ and uncertainties using frequencies extracted from the lineshapes with a variety of fitting procedures (Sec. IV C). The uncertainty in $g / 2$ is increased to cover the range of values that come from various fits since the slight broadening of the observed lineshapes is not yet understood (Sec. IV D). The lineshape uncertainty is the largest uncertainty contribution to the $g / 2$ for the most precise of our measurements.

\section{A. Lineshape model}

Detailed discussion of the cyclotron and anomaly resonance lineshapes are available [29,44,52]. Here we give a summary, stressing what must be assumed and calculated to enable the measurement of the electron magnetic moment and the uncertainties in the measurement.

For cyclotron and anomaly drives left on for a time much longer than the inverse-linewidth and inverse-axial-damping rate $\gamma_{z}^{-1}$, the probability $P$ for a transition to occur after a time $T$ is [52]

$$
P=\frac{1}{2}\left\{1-\exp \left[-\pi T \Omega^{2} \chi(\omega)\right]\right\},
$$

which depends on the Rabi frequency $\Omega$ and the lineshape function $\chi(\omega)$ and saturates at $1 / 2$ for strong drives. The radiative decay of cyclotron excitations reduces the cyclotron lineshape saturation value. For a delay $t$ between the end of the drive and the beginning of the state measurement, the lineshape saturates at $\exp \left(-\gamma_{c} t\right) / 2$.

In general, the lineshape $\chi(\omega)$ is the Fourier transform of a correlation function $\tilde{\chi}(t)$, which is related to the statistical average of any fluctuations in the magnetic field, $\omega(t)[52]$ :

$$
\tilde{\chi}(t)=\left\langle\exp \left[-i \int_{0}^{t} d t^{\prime} \omega\left(t^{\prime}\right)\right]\right\rangle .
$$

\section{During quantum jump spectroscopy_Brownian axial motion}

The same magnetic bottle that makes a QND coupling of the cyclotron and spin energies to the axial frequency couples the axial energy to the cyclotron and anomaly frequencies and 
is the primary source of the observed lineshape. For an electron on axis $(\rho=0)$, it adds a $z^{2}$ dependence to the magnetic field and thus to the cyclotron and anomaly frequencies, here collectively $\omega$ :

$$
\omega(z)=\omega_{0}\left(1+\frac{B_{2}}{B} z^{2}\right) .
$$

When probing the cyclotron or anomaly resonance for quantum jump spectroscopy, the axial motion is in thermal equilibrium with the detection amplifier circuit, since the amplifier power is turned off. Thus, the axial position $z$ undergoes Brownian motion. The lineshape $\chi(\omega)$ is a statistical average of these Brownian fluctuations and takes various forms depending on the relative lengths of the fluctuation timescale - the inverse-axial-damping time $\gamma_{z}^{-1}$-and the inverse-linewidth coherence time. This linewidth roughly corresponds to the frequency shift at the root-mean-square thermal axial amplitude,

$$
\Delta \omega=\omega_{0} \frac{B_{2}}{B} z_{\mathrm{rms}}^{2}=\omega_{0} \frac{B_{2}}{B} \frac{k T_{z}}{m \omega_{z}^{2}} .
$$

The contribution of inhibited cyclotron decay width to the cyclotron linewidth (discussed below) is much smaller, but is still significant for the anomaly linewidth.

The relevant correlation function $\tilde{\chi}(t)$ is found by inserting Eq. (24), whose time-dependence is in the axial Brownian motion, into Eq. (23) to get

$$
\tilde{\chi}(t)=e^{-i \omega_{0} t}\left\langle\exp \left[-i \omega_{0} \frac{B_{2}}{B} \int_{0}^{t} d t^{\prime} z\left(t^{\prime}\right)^{2}\right]\right\rangle .
$$

Taking the statistical average gives three equivalent solutions for the lineshape [52],

$$
\begin{aligned}
& \chi(\omega)=\frac{4}{\pi} \operatorname{Re}\left[\gamma^{\prime} \gamma_{z} \int_{0}^{\infty} d t \frac{e^{i\left(\omega-\omega_{0}\right) t} e^{-\frac{1}{2}\left(\gamma^{\prime}-\gamma_{z}\right) t} e^{-\frac{1}{2} \gamma_{c} t}}{\left(\gamma^{\prime}+\gamma_{z}\right)^{2}-\left(\gamma^{\prime}-\gamma_{z}\right)^{2} e^{-\gamma^{\prime} t}}\right] \\
= & \frac{4}{\pi} \operatorname{Re}\left[\frac{\gamma^{\prime} \gamma_{z}}{\left(\gamma^{\prime}+\gamma_{z}\right)^{2}} \sum_{n=0}^{\infty} \frac{\left(\gamma^{\prime}-\gamma_{z}\right)^{2 n}\left(\gamma^{\prime}+\gamma_{z}\right)^{-2 n}}{\left(n+\frac{1}{2}\right) \gamma^{\prime}+\frac{1}{2}\left(\gamma_{c}-\gamma_{z}\right)-i\left(\omega-\omega_{0}\right)}\right] \\
= & -\frac{4}{\pi} \operatorname{Re}\left[\frac{\gamma_{z}}{K\left(\gamma_{z}+\gamma^{\prime}\right)^{2}} F_{1}\left(1,-K ; 1-K ; \frac{\left(\gamma_{z}-\gamma^{\prime}\right)^{2}}{\left(\gamma_{z}+\gamma^{\prime}\right)^{2}}\right)\right],
\end{aligned}
$$

where

$$
\begin{gathered}
\gamma^{\prime}=\sqrt{\gamma_{z}^{2}+4 i \gamma_{z} \Delta \omega}, \\
K=\frac{2 i\left(\omega-\omega_{0}\right)+\gamma_{z}-\gamma^{\prime}-\gamma_{c}}{2 \gamma^{\prime}},
\end{gathered}
$$

"Re" denotes the real part, and ${ }_{2} F_{1}(a, b ; c ; z)$ is a hypergeometric function. Examples of the lineshape for various values of $\gamma_{z} / \Delta \omega$ and $\gamma_{z} / \gamma_{c}$ are shown in Fig. 9.

In contrast to some previous presentations of the lineshape $[29,44]$, we have not taken the limit of low cyclotron damping. ${ }^{1}$

\footnotetext{
${ }^{1}$ Brown does keep a nonzero cyclotron damping rate when deriving the anomaly line, with a result ([52], Eq. 6.12) identical to that
}

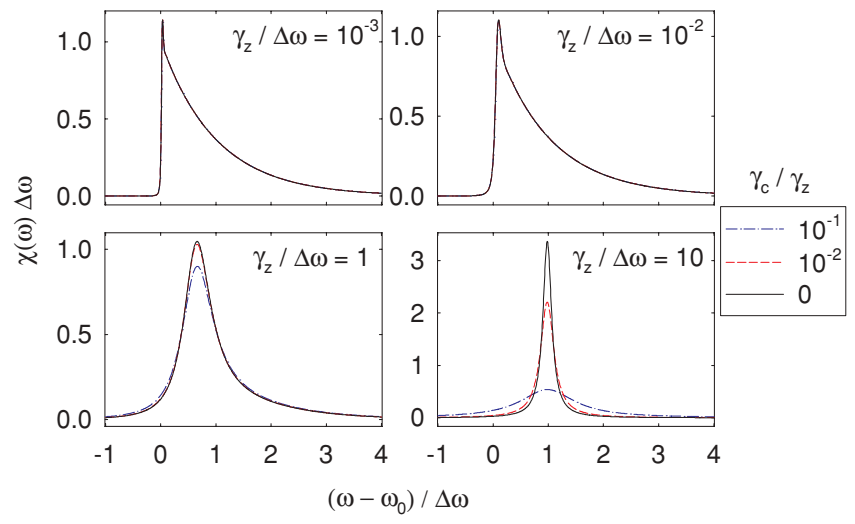

FIG. 9. (Color online) The theoretical Brownian-motion lineshape for various $\gamma_{z} / \Delta \omega$ and $\gamma_{c} / \gamma_{z}$. Our cyclotron line has $\gamma_{z} / \Delta \omega \approx$ $10^{-2}$. Our anomaly line has $\gamma_{z} / \Delta \omega \approx 10$. For our $\gamma_{z}, \gamma_{c} / \gamma_{z}=10^{-1}$ $10^{-2}$ correspond to lifetimes of $1.6 \mathrm{~s}-16 \mathrm{~s}$. The infinite-lifetime limit is $\gamma_{c} / \gamma_{z}=0$.

Our resolution of the anomaly line is fine enough that we must include the broadening from the finite lifetime of the $\left|1,-\frac{1}{2}\right\rangle$ state.

The cyclotron and anomaly lines are in two limits of this lineshape. For the cyclotron line, $\gamma_{z} / \Delta \omega \approx 10^{-2} \ll 1$ and the axial motion is essentially decoupled from the amplifier during the inverse-linewidth coherence time. During that time, the electron retains its axial oscillation amplitude. The lineshape is a Lorentzian with the natural linewidth, $\gamma_{c}$, and centered on the frequency given by Eq. (24) with the rms axial amplitude of that state as $z$. We do not know the axial amplitude, however, and since excitation attempts occur on timescales longer than $\gamma_{z}^{-1}$, subsequent attempts will have different axial amplitudes. The composite lineshape after many attempts is thus the convolution of the instantaneous lineshape (the narrow Lorentzian) and the Boltzmann distribution of axial oscillation amplitudes. That is, the lineshape is a decaying exponential with a sharp edge at $\omega_{0}$ and a width of $\Delta \omega$. The cyclotron line is close to this "exponential" limit and should have a sharp edge at the zero-axial-amplitude cyclotron frequency that is useful for quick field measurements such as tracking drifts.

In contrast, the anomaly line has $\gamma_{z} / \Delta \omega \approx 10 \gg 1$. The axial motion is strongly coupled to the amplifier on a time scale corresponding to the linewidth coherence time, during which time the axial amplitude averages to the thermal $z_{\text {rms. }}$ The resulting lineshape approaches a natural-linewidth Lorentzian offset from $\omega_{0}$ by $\Delta \omega$ through a Lorentzian with a width of $\gamma_{c}+2 \Delta \omega^{2} / \gamma_{z}$.

\section{During cyclotron lifetime measurements-driven axial motion}

The axial motion is in thermal equilibrium with the detection circuit during the resonance probes of a $g / 2$ measurement, but it is necessarily driven during QND detection. Probing the lineshape with this detection drive on adds a coherent oscillation of $z$ to the Brownian motion considered above. The

presented here. The derivation assumes an anomaly excitation technique that differs from ours (Sec. III I), but the only effect is a redefinition of the Rabi frequency [51]. 
magnetic bottle coupling again translates this motion into a lineshape that depends on the amplitude of the axial oscillation. In the weak-coupling limit $\left(\gamma_{z} / \Delta \omega \ll 1\right)$ that corresponds to our cyclotron line, the lineshape is ([52], Eq. 7.18)

$$
\begin{aligned}
\chi_{d}(\omega) & =I_{0}\left(\frac{2 \sqrt{\left(\omega-\omega_{0}\right) \Delta_{d} \omega}}{\Delta \omega}\right) \theta\left(\omega-\omega_{0}\right) \\
& \times \frac{1}{\Delta \omega} \exp \left(-\frac{\omega-\omega_{0}+\Delta_{d} \omega}{\Delta \omega}\right),
\end{aligned}
$$

where

$$
\Delta_{d} \omega=\omega_{0} \frac{B_{2}}{B} \frac{A^{2}}{2},
$$

$A$ is the driven axial amplitude, $\theta(x)$ is the Heaviside step function, and $I_{0}(x)$ is the order-zero modified Bessel function. We make extensive use of this driven lineshape in calibrating the axial oscillation amplitude in Sec. VD.

\section{Other magnetic field fluctuations}

Although the cyclotron line is in the exponential lineshape limit $\left(\gamma_{z} / \Delta \omega \ll 1\right)$ and should have a sharp low-frequency edge, all our data show an edge width of $0.5-1 \mathrm{ppb}$ (Fig. 8). We model this discrepancy as additional fluctuations in the magnetic field. (Some potential sources are discussed at the end of this section.) Such fluctuations can be described by adding a noise term $\eta(t)$ to the Brownian motion axial fluctuations of Eq. (24),

$$
\omega(t)=\omega_{0}\left(1+\frac{B_{2}}{B} z(t)^{2}+\eta(t)\right),
$$

so that the lineshape is given by the Fourier transform of

$$
\tilde{\chi}(t)=\left\langle\exp \left[-i \int_{0}^{t} d t^{\prime} \omega_{0}\left(1+\frac{B_{2}}{B} z\left(t^{\prime}\right)^{2}+\eta\left(t^{\prime}\right)\right)\right]\right\rangle .
$$

For magnetic field noise that is not correlated with the axial fluctuations, the average factors into

$$
\begin{aligned}
\tilde{\chi}(t)= & e^{-i \omega_{0} t}\left\langle\exp \left[-i \omega_{0} \frac{B_{2}}{B} \int_{0}^{t} d t^{\prime} z\left(t^{\prime}\right)^{2}\right]\right\rangle \\
& \times\left\langle\exp \left[-i \omega_{0} \int_{0}^{t} d t^{\prime} \eta\left(t^{\prime}\right)\right]\right\rangle .
\end{aligned}
$$

The first two factors are the Brownian-motion lineshape and the third is an additional noise broadening. Because of the Fourier transform convolution theorem, the resulting noisy lineshape is the noise-free lineshape of Eq. (27) convolved with a noise function.

\section{Invariance of the mean frequency}

An important feature of the Brownian-motion lineshape, which we use in our primary line-splitting technique, is the independence of its mean and $\gamma_{z}$. For the low drive strengths used in this measurement, we may expand Eq. (22) to lowest order,

$$
P=\frac{1}{2} \pi T \Omega^{2} \chi(\omega),
$$

such that the excitation probability is linear in the lineshape function. Then, the average frequency of the lineshape, $\langle\omega\rangle=\int_{-\infty}^{\infty} \omega \chi(\omega) d \omega$, always corresponds to that given by the thermal $z_{\mathrm{r} m s}$ in the magnetic bottle field [Eq. (24)]. That is ([52], Eq. 1.29),

$$
\langle\omega\rangle=\omega_{0}+\Delta \omega
$$

This mean is easily verified in the exponential and Lorentzian limits described above, but applies for all $\gamma_{z} / \Delta \omega$.

Importantly, any additional magnetic field noise $\eta$ affects both cyclotron and anomaly lines identically [Eq. (34)]. If the noise fluctuates symmetrically, that is, the mean frequency of the noise function is zero, then the mean frequencies of the lines are unchanged. A nonzero noise function average would shift both lines by proportionally the same amount; this shift would then cancel in the calculation of $g / 2$ [Eq. (10)].

Since the large-drive saturation of Eq. (22) invalidates this mean-frequency invariance, the excitation probabilities are kept below $20 \%$ to keep saturation shifts of the mean frequency smaller than the statistical uncertainty.

\section{B. The weighted mean method to extract frequencies from lineshapes}

As justified above, the preferred method is to use the lineshape-weighted mean values of the cyclotron and anomaly frequencies in Eq. (10). Insofar as the two frequencies are essentially proportional to the magnetic field, any effective fluctuations in the magnetic field would average the two frequencies in much the same way.

In detail, we extract $\bar{f}_{c}$ and $\bar{v}_{a}$ from their resonance lines by binning the excitation attempts into histograms and calculating the weighted-mean frequency of each line using a trapezoidrule integration. The uncertainty in determining $\bar{f}_{c}$ and $\bar{v}_{a}$ comes from binomial uncertainties in the number of successes in each histogram bin, and we assign it as the "statistical" uncertainty.

The assumptions for the weighted-mean method are unsaturated lines as well as identical temperature and drive conditions during cyclotron and anomaly excitations. A further assumption is a fluctuation spectrum that either is symmetric (and thus does not shift the means) or is identical during cyclotron and anomaly excitations (and thus gives shifts that cancel in the calculation of $g / 2$ ).

The values of $g / 2$ that are the result of our weighted mean procedure, for four different values of the magnetic field, are reported in Table V. We defer further discussion of these values until we first discuss the statistical and then the systematic uncertainties in the following sections.

\section{Lineshape model uncertainties}

To test these assumptions, and to check the uncertainties, we use maximum-likelihood fits of the data to a lineshape that includes a specific model of the field-noise spectrum: the Brownian-motion lineshape convolved with a Gaussian whose width is left as a fit parameter. These fits determine the zero-axial-amplitude $\bar{f}_{c}$ and $\bar{v}_{a}$, which are used in Eq. (10) to calculate $g / 2$. The agreement between this line fit $g / 2$ and that from the weighted mean is our primary check on the lineshape model, and quantifying this agreement provides a systematic "lineshape" uncertainty. 
Specifically, we compare the agreement of two weightedmean and four fitted determinations of $g / 2$. Because the weighted-mean method requires binning the data into a histogram, we check that our result is independent of bin width by using two different numbers of bins (50 and 100) at each field. We conduct four maximum-likelihood fits to the data. The first fit treats each excitation attempt separately and fits the two lines sequentially-cyclotron then anomaly. The second is a sequential fit to histogrammed data. The third is a simultaneous fit of both cyclotron and anomaly lines to histogrammed data. The fourth is a sequential fit to histogrammed data with reduced axial damping.

Each fit involves six free parameters. There are the cyclotron and anomaly frequencies, the two corresponding Rabi frequencies, the axial temperature, and the width of the convolved Gaussian. Fitting in four different ways gives different sensitivities to the fit parameters. In three cases, we use sequential fits of the two lines-cyclotron then anomalybecause the cyclotron line better differentiates between the two main broadening mechanisms: axial temperature and the Gaussian noise function. Both broaden the anomaly line symmetrically. The cyclotron line is broadened symmetrically by the Gaussian noise function and asymmetrically by thermal axial motion. We include one fit with a reduced axial damping because $\gamma_{z}$ is difficult to measure precisely. The fits are much faster when using histogrammed data, but we include one with no binning to eliminate a possible binning systematic.

At each field, we thus have six analyses of the $g$ value. We quantify their agreement as a " $g$-value range" by taking half the difference between the lowest and highest error bars (one-standard-deviation confidence) of the six. We treat the 50-bin weighted-mean result as the measurement of $g / 2$ and its uncertainty as the statistical uncertainty. The difference (in quadrature) between the $g$-value range and the statistical uncertainty is assigned as a "lineshape model" uncertainty. To be cautious, we avoid a reduction of the lineshape model uncertainty when averaging the $g / 2$ measurements at four magnetic fields by treating the lowest uncertainty (that at 149.2 GHz) as a correlated one. It corresponds to our best understanding of the lineshape model. Any additional uncertainty is added as an uncorrelated uncertainty. Thus, at each field the original $g$-value range is broken into three uncertainties and is equal to their sum in quadrature. These uncertainties are summarized in Table V.

Figure 8 displays the data set used to determine the anomaly and cyclotron frequencies, with 37 nighttime runs at four magnetic fields. The data, binned into histograms (points), fit well to a convolution (solid curve) of a Gaussian resolution function (solid inset curve) and a Brownian-motion lineshape (dashed curve), as indicated by one-standard-deviation confidence limits for distributions of measurements about the fits (gray bands). The vertical lines indicate the weighted-mean frequencies and their uncertainties from both statistics and lineshape model. An additional probe of the broadening comes from histograms (inset) of the edge-tracking data, which is used for drift normalization as described in Sec. III J. Although the precise distribution of this data depends on the details of the edge-tracking procedure, simulations of our procedure indicate that it should be distributed with a width comparable to- - within a factor of two of - the Gaussian broadening width. As shown in Fig. 8, the two agree well.

The resonance lines at $147.5 \mathrm{GHz}$ and $151.3 \mathrm{GHz}$ are broader than those at the other two fields, consistent with a higher axial temperature. The broader lines naturally contribute more lineshape uncertainty to the determination of $g / 2$. The temperature differences are also not understood, and we have not been able to identify corresponding differences in the measurement procedure used at the four field values. In fact, whether it is noise, residual heat in the amplifier circuit, or some other effect that makes $T_{z}$ higher than the trap temperature is also not yet understood.

The weighted-mean and line-fit methods should yield a $g / 2$ that is independent of axial temperature. Nevertheless, we check for any systematic trends related to axial temperature by taking an additional set of data at $149.2 \mathrm{GHz}$ with the refrigerator operating at $500 \mathrm{mK}$ instead of $100 \mathrm{mK}$. Whereas the $100 \mathrm{mK}$ data fit to $T_{z}=0.23(3) \mathrm{K}$, the $500 \mathrm{mK}$ data fit to $0.55(2) \mathrm{K}$, consistent with our deliberate heating. The highertemperature weighted-mean calculation has a statistical uncertainty of $0.30 \mathrm{ppt}$, and the maximum-likelihood-fit checks give an uncorrelated lineshape model uncertainty of $0.46 \mathrm{ppt}$, both larger than those of the lower-temperature data at $149.2 \mathrm{GHz}$ in agreement with the temperature-uncertainty correlation noted above. Including the statistical and uncorrelated lineshape model uncertainties, the difference between the $149.2 \mathrm{GHz}$, $500 \mathrm{mK} g / 2$ and the $100 \mathrm{mK} g / 2$ is $0.5(6) \mathrm{ppt}$, which is consistent with zero.

\section{Possible broadening sources}

What could cause the additional line-broadening? We have modeled this effect above as fluctuations in the magnetic field. Attributing the line broadening to field noise assumes that the fluctuation timescale is not so fast that the noise averages away during an excitation attempt. The relevant comparison timescale is the inverse-linewidth coherence time (200 $\mu$ s for the cyclotron line and $200 \mathrm{~ms}$ for the anomaly line), and any line-broadening noise must fluctuate near to or slower than these timescales. Noise broadening from slow fluctuations is analogous to the exponential limit $\left(\gamma_{z} / \Delta \omega \ll 1\right)$ of the noise-free cyclotron line, which takes its shape from the long axial fluctuation time and the distribution of axial energies. Our edge-tracking data provide an upper timescale of minutes for the noise timescale because we see no correlation between adjacent edge-tracking points, which come at intervals of several minutes. This range of allowed timescales constrains the possible fluctuation mechanisms.

We have considered and ruled out several possible sources. Section III D includes several known producers of field noisethe local subway, cryostat pressure changes, ambient temperature changes, and temperature-dependent paramagnetismand our efforts to reduce them. Phase noise on the cyclotron and anomaly drives would mimic field fluctuations, though the similar broadening observed on each line is not what one would expect given the vastly different drive frequencies. Estimates based on the microwave signal generator's specified phase noise and additional noise from an ideal multiplier suggest any frequency deviations should be over two orders of magnitude below the level required to explain the cyclotron broadening. 
Remaining candidates include relaxation of stresses in the solenoid windings or vibration of the trap electrodes in an inhomogeneous magnetic field. Even with the magnetic field tuned to its specified homogeneity, a $100 \mu \mathrm{m}$ motion of the trap electrodes would cause a $0.1 \mathrm{ppb}$ field variation. Such a motion could be caused by vibrations that drive the dilution refrigerator like a 2.2-m-long pendulum.

One additional source of broadening is a distribution of magnetron radii in the $-\rho^{2} \hat{\mathbf{z}} / 2$ portion of the magnetic bottle [Eq. (19)]. Ideally, our sideband cooling procedure will produce a thermal distribution of magnetron energies with a temperature related to the axial temperature by [29]

$$
T_{m}=-\frac{\bar{v}_{m}}{\bar{v}_{z}} T_{z} .
$$

(The negative sign indicates that the magnetron degree of freedom is unbound.) Such an ideal distribution would broaden the cyclotron line by $\bar{v}_{m} / \bar{v}_{z}$ times the axial broadening $\Delta \omega$, which is $10^{2}-10^{3}$ times too small to account for the observed width. Nevertheless, we have checked that the broadening is not due to a thermal distribution of magnetron energies by deliberately sideband cooling with the amplifiers on $\left(T_{z}=\right.$ $5 \mathrm{~K}$ ) before damping the axial energy to $230 \mathrm{mK}$; we found no change in the broadening. Sideband cooling with the coherent distribution of axial states found during self-excitation would produce a coherent distribution of magnetron states with a width that could explain the broadening, but the electron is not self-excited during sideband cooling. Interestingly, prior studies of sideband cooling could only achieve cooling to energies 400 times the ideal limit [29]. If this nonideal energy limit corresponds to a distribution of energies as well, it could explain the observed broadening; though, it is unclear what mechanism limits the cooling and whether it applies to our setup as well.

Our knowledge of the lineshape model provides the largest uncertainty in recent $g / 2$ measurements [1,2]. Future efforts will focus on explaining or eliminating the lineshape broadening.

\section{CAVITY CONTROL}

\section{A. Overview}

The averaging time needed to observed one-quantum cyclotron transitions is obtained by inhibiting [42,49] the spontaneous emission of synchrotron radiation. Inhibition by factors of 100 and more is accomplished with a cylindrical Penning trap cavity that was invented for this purpose [6]. The cylindrical trap shapes the radiation field within the interior of a conducting right circular cylinder, modifying the density of radiation states in a way that can be studied and understood, at the same time as it provides the high quality electrostatic quadrupole potential needed to detect one trapped electron with good signal-to-noise [7].

The extremely useful inhibition of spontaneous emission comes at a cost insofar as coupled oscillators-the cyclotron oscillator and the radiation mode oscillators-pull each other's frequency. The challenge that thus arises is that the interaction of the electron and the cavity modes also shifts the cyclotron frequency $[1,53]$. Typical cavity shifts are at the ppt-level in the cyclotron frequency and ppb-level in the anomaly frequency-large enough to unacceptably shift the value of $g / 2$. The cylindrical trap geometry was selected to provide familiar and well understood boundary conditions within which the properties of the radiation field, and hence the shifts that these fields produce, could be calculated and understood.

The Penning trap establishes the boundary conditions of a right circular cylinder for microwaves within the trapdetermining the electric and magnetic field of the transverse electric and magnetic modes. Of course, the boundaries are not perfect because the electrodes are deliberately slit so that sections of the cavity can be separately biased trap electrodes, because the electrodes contract as the trap is cooled from 300 to $0.1 \mathrm{~K}$, and the electrodes are not perfectly machined and aligned. The result is that the resonant frequency and damping factor for each radiation mode are slightly shifted and must be measured within the cold trap cavity.

We used two independent methods to investigate the radiation modes of the cylindrical trap cavity in situ. For our 2006 measurement, the synchronization of the collective motion of many electrons [8,9] was used to trace out the cyclotron damping rate as a function of the electron cyclotron frequency, allowing us to identify and label the transverse electric and transverse magnetic modes. For the 2008 measurement, the one-electron cyclotron damping rate was directly measured as a function of both the cyclotron frequency and the amplitude of the axial oscillation through the standing wave field of the cavity modes. We demonstrate a 165(4) $\mu \mathrm{m}$ axial offset between the electrostatic center of the trap and the center as defined by the standing wave fields, and place a limit of $\rho<10 \mu \mathrm{m}$ on any radial offset.

The electric and magnetic fields of these modes are used as input for an analytic calculation that is properly renormalized to avoid self-energy infinities [10,54]. The calculation is then adapted in a semiempirical way to better describe the way that the electron cyclotron frequency is shifted and damped. For the 2006 measurement we were able to reduce the cavity shift uncertainty to $0.39 \mathrm{ppt}$ [1]. For the 2008 measurement the cavity shift uncertainty was reduced by an additional factor of six, to 0.06 ppt [2].

\section{B. Mode detection with synchronized electrons}

Our first technique for probing the radiation modes of the not-quite-ideal trap cavity uses the synchronized axial motion of approximately $2 \times 10^{4}$ electrons trapped near its center. When we first developed this method [8], we were able to use it to identify more than 100 radiation mode of a cylindrical trap cavity, with frequencies between $20 \mathrm{GHz}$ and $160 \mathrm{GHz}$, with those of an ideal right circular cylinder. The cavity used then was nearly identical to the one used for the 2006 and 2008 measurements. The method seemed ideal in that it produced in situ a spectrum with peaks at the frequency of radiation modes (Fig. 10), and with lineshapes that were Lorentzian and independent of the number of electrons used (as long as saturation and strongly coupled regimes were avoided). We thus interpreted these widths as the inverse of the mode quality or $Q$ factors.

The electrons are excited by modulating the potential applied to the bottom endcap electrode at a frequency that is nearly twice the axial oscillation frequency of the electrons, 


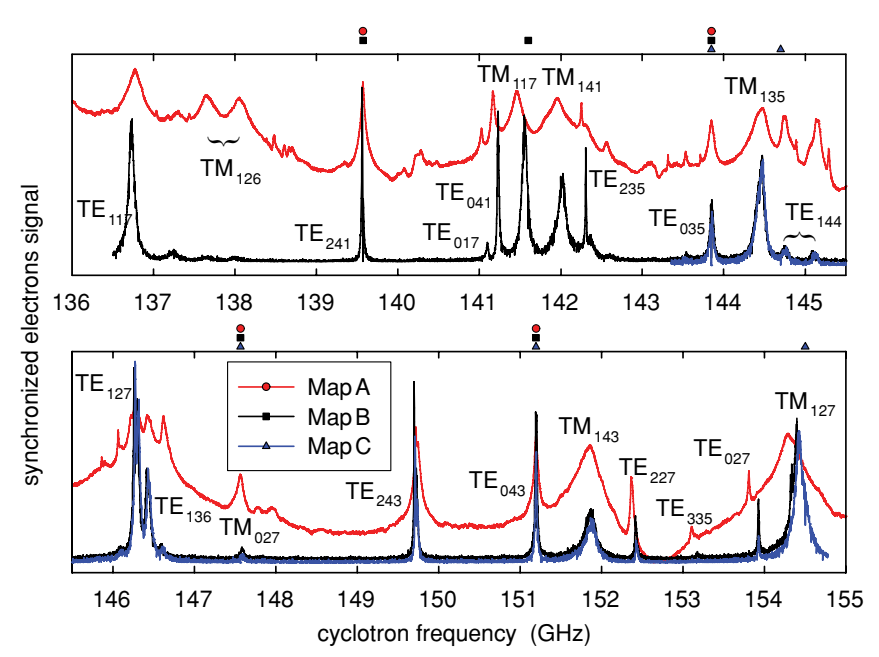

FIG. 10. (Color) Modes of the trap cavity as observed with synchronized electrons in three separate sweeps. The calibration frequencies are indicated with symbols above the plots. The striking difference between maps B and C and map A is discussed in the text.

$2 \bar{v}_{z}$. This parametric modulation of the trapping potential heats the electrons. The axial oscillation of their center-of-mass grows exponentially but this growth is limited to a steady-state value because the trapping potential is anharmonic for large oscillation amplitudes. Our prior work [8,9] showed that the measured oscillation amplitude was large when the cyclotron damping rate was high - when the cyclotron frequency of the electrons was resonant with a radiation mode in Fig. 10. The shape of the spectrum was remarkably independent of the number of electrons $N$, which could be varied to change the cyclotron damping rate of the center-of-mass motion $N \gamma_{c}$. In the strong coupling regime, where $N \gamma_{c}$ exceeded the damping rate of the cavity modes, the observed peaks split into two. The axial motion of the electrons also generates motional sidebands in the observed cavity spectra. The method was very robust and reproducible.

We learned a great deal about how this method worked by varying the number of electrons used from $10^{5}$ electrons [8,9] down to only 2 [55], and by varying electrostatic anharmonicity, the parametric drive strength and the slow rate at which the cyclotron frequency swept through resonance with the radiation modes. For electrons driven at twice their preferred oscillation frequency, the resulting axial oscillations can have either of two oscillation phases that differ by $\pi$. Between resonances with the cavity radiation modes, where the cyclotron damping was very small, the electrons oscillated in equal numbers with both of the two axial oscillation phases, so that the detected signal from the axial center-of-mass is very small. On resonance with the cavity radiation modes, with very strong cyclotron center-of-mass damping, the electrons synchronize into axial oscillations with predominately one of the two possible phases, producing a large detected signal.

This method is not yet fully understood despite boundary conditions that are very carefully controlled. We have written down what we believe are the full equations of motion for the $N$ electrons [9], but have not obtained a solution that represents the simple and striking behavior that is observed. One additional clue may be that the method worked robustly over a long time in an apparatus for which the trap's vacuum enclosure and the detection electronics were submerged in liquid helium. In a brief trial, we did not succeed in getting robust performance in an apparatus in which the trap vacuum container and the detection electronics were cooled by thermal contact to a liquid helium dewar. Perhaps the temperature and noise of the detectors are more important than initially supposed.

In the apparatus used for the $g / 2$ measurements, cooled to $0.1 \mathrm{~K}$ rather than to $4.2 \mathrm{~K}$ and with improved detection electronics, the interpretation of these cavity mode spectra has become much less clear. For causes that we have not yet discerned, we sometimes measure spectra that are like what we measured in the first studies of the cavity spectra [9] (blue and black traces in Fig. 10). However, much more often we measure severely broadened spectra (red trace in Fig. 10).

The observed cavity spectra nonetheless give us an important in situ probe into the spectrum of the cavity radiation modes. It takes several hours to measure a spectrum. In light of the striking difference (mentioned above and discussed in more detail below) the broadened spectra marked A in Fig. 10 is not used in the cavity shift analysis. The 2008 measurements uses the same trap cavity and measured spectra as in 2006 [1], but our understanding has improved due to the addition of a new probe of the cavity spectra (next section) and through more thorough analysis.

The frequency axis for the cavity spectra is calibrated during the sweep by applying a strong cyclotron drive at discrete frequencies (symbols in Fig. 10). When resonant with $\bar{v}_{c}$, the magnetic-bottle coupling shifts $\bar{v}_{z}$ and the parametric drive is no longer resonant, producing a sharp dip in the signal such as that on $\mathrm{TE}_{241}$ near $139.6 \mathrm{GHz}$. The cyclotron frequencies for the remainder of the map are assigned with a linear interpolation function between these discrete calibration points. (Map A is calibrated with an earlier technique that involved stopping the magnetic field where marked and measuring $\bar{v}_{c}$ directly.) Comparing the mode frequencies with those of an ideal cylinder with dimensions similar to the trap dimensions allows us to identify and label the TE and TM modes. [9].

Three features aid the interpretation of the cavity spectra

(1) Strong cloud-mode coupling can split the Lorentzian response into a pair of normal modes. The $Q$ of the split peaks is higher than that of the mode, making it difficult to estimate the mode $Q$ from parametric mode maps alone.

(2) The large axial motion of the cloud during the measurement (limited only by trap anharmonicity) probes the electric-field profiles of the modes and amplitude-modulates $\gamma_{c}$, producing axial-frequency sidebands at $\pm \bar{\nu}_{z}$ for modes with a node at the trap center (e.g., $\mathrm{TE}_{144}$ at $\left.145.0 \mathrm{GHz}\right)$ and $\pm 2 \bar{v}_{z}$ for modes with an antinode at the trap center. These motional sidebands are further explored below for the single-electron case.

(3) Nonzero cloud size or a relative offset between the electrostatic center and the mode center allows coupling to modes with nodes at the mode center (e.g., $\mathrm{TE}_{136}$ at $146.4 \mathrm{GHz}$ and $\mathrm{TE}_{243}$ at $149.7 \mathrm{GHz}$ ). 
TABLE II. Comparison of the mode parameters from the multielectron parametric mode maps and the single-electron lifetime fits.

\begin{tabular}{lccc}
\hline \hline & & parametric & lifetime \\
\hline $\mathrm{TE}_{127}$ & $v_{c}(\mathrm{GHz})$ & $146.289(7)$ & $146.322(13)$ \\
& $Q$ & $4600(900)$ & $4900(300)$ \\
$\mathrm{TE}_{136}$ & $v_{c}(\mathrm{GHz})$ & $146.436(7)$ & $146.415(2)$ \\
& $Q$ & $2200(60)$ & $4800(200)$ \\
$\mathrm{TM}_{143}$ & $v_{c}(\mathrm{GHz})$ & $151.865(4)$ & $151.811(16)$ \\
& $Q$ & $890(10)$ & $1270(70)$ \\
\hline \hline
\end{tabular}

For the four magnetic fields at which we measure $g / 2$, the modes with the largest influence on the cavity shift are $\mathrm{TE}_{127}$, $\mathrm{TE}_{136}$, and $\mathrm{TM}_{143}$. Table II summarizes the mode frequencies and $Q$ values obtained from Lorentzian fits to the cavity spectra $\mathrm{B}$ and $\mathrm{C}$ of Fig. 10 after accounting for any normal-mode splitting. The listed uncertainties are what is estimated as part of the nonlinear least squares fitting to Lorentzian peaks, and does not include any additional systematics contribution.

In light of the differences between the cavity spectra measured when this method was first developed, and what we now are able to observe, and because we are not currently always able to robustly produce such spectra, we have carefully reflected upon our measurement technique. Early studies on mode detection with synchronized electrons [9] found the center-of-mass amplitude closely followed $\gamma_{c}$ for wide ranges of parameters, yielding Lorentzian mode profiles except in cases of normal-mode splitting or motional sidebands. With our current apparatus we find three classes of behavior:

(1) Convincing cavity spectra with the detected center-ofmass signal tracking $\gamma_{c}$ with Lorentzian mode profiles and no center-of-mass motion far from modes, as seen in B and C of Fig. 10.

(2) Broadened cavity spectra in which the signal never disappears but increases and decreases with $\gamma_{c}$, as seen in A.

(3) No cavity spectrum at all. With nominally the same parameters as for the previous two cases we do not see the detected signal change as the magnetic field is varied to sweep the cyclotron frequency. This is the most common behavior.

There are substantial differences between the earlier apparatus (Ref. [9]) and that used for the 2006-2008 measurements, even though the trap electrodes themselves are essentially the same.

(1) 40-times lower cavity temperature should increase the interparticle Coulomb interaction and enhance collective motion [56,57].

(2) 10-times deeper axial potential should have a similar effect.

(3) Improved detection electronics [44] should offer greater detection sensitivity while producing less noise to heat the trapped electrons.

(4) 10-times larger electron clouds are used because we find that smaller clouds rapidly saturate, with all electrons in one of the two oscillation phases.

(5) More heavily filtered and noise-free electrical environment should reduce noise driven transitions between the bistable states.

The three maps of Fig. 10 were taken using the same trap cavity over the course of 18 mos, during which time the electrodes were thermally cycled to room temperature several times, the refrigerator was inserted and removed, and the magnet was quenched with the electrodes inside. At no point were the electrodes themselves disassembled or adjusted. The general alignment of the features and the precise alignment of the calibration points in Fig. 10 indicate that the trap cavity and its resonant modes are robust against stresses and thermal cycles. Any misalignments in the location of a particular mode may be attributed to the calibration process, specifically to nonlinear charging rates from the power supplies, rather than to real shifts in the mode frequencies. This consistency suggests that the cavity itself is stable, though the variety of parametric behaviors discussed in the previous paragraph motivates our use of an independent, one-electron mode detection technique.

\section{Cavity coupling to a single electron}

\section{Overview}

The expressions describing the coupling of a single electron to the electromagnetic modes lie at the heart of our cavity analysis. Their importance is twofold: First, they are required for the calculation of the cyclotron frequency shifts, $\Delta \omega_{c}$, and second, measurements of the cyclotron damping rate, $\gamma_{c}$, allow a characterization of the cavity mode structure independent of the multi-electron technique of the previous section. In this section, we present two formulas for the cavity-induced cyclotron frequency shift and damping rate. They differ in their treatment of the electron's axial motion. Equation (40) applies for an electron with negligible axial motion, corresponding to the cold thermal axial distribution during cyclotron or anomaly excitation. Equation (58) applies for a larger axial motion that begins to probe the mode's standing wave, corresponding to the self-excited axial state during detection and measurement of $\gamma_{c}$.

\section{Single-mode approximation}

Before beginning a full calculation of the cyclotron motioncavity coupling, it is worth modeling the interaction between the electron and a single nearby mode, here denoted $\mathrm{M}$, to give an indication of the character of the electron-mode coupling. This approximation will eventually aid in modeling the coupling including axial motion in Sec. V C 5. The interaction may be approximated as that of two coupled oscillators with the resulting electron frequency shift and damping rate given by [10]

$$
\begin{aligned}
\Delta \omega_{c} & =\frac{\gamma_{\mathrm{M}}}{2} \frac{\delta}{1+\delta^{2}}, \\
\gamma_{c} & =\gamma_{\mathrm{M}} \frac{1}{1+\delta^{2}} .
\end{aligned}
$$

Here, $\gamma_{M}$ is the cyclotron damping rate when the electron is exactly resonant with the mode and $\delta$ is the relative detuning, defined as

$$
\delta=\frac{\bar{\omega}_{c}-\omega_{\mathrm{M}}}{\Gamma_{\mathrm{M}} / 2} .
$$

The mode full-width at half-maximum, $\Gamma_{\mathrm{M}}$, arises because of losses in the cavity and may be written in terms of a quality factor, $Q_{\mathrm{M}}$, with the definition: $Q_{\mathrm{M}}=\omega_{\mathrm{M}} / \Gamma_{\mathrm{M}}$. The 
cyclotron frequency is maximally shifted by $\pm \gamma_{\mathrm{M}} / 4$ at $\delta= \pm 1$. Furthermore, provided the cyclotron frequency is detuned far enough from a mode that $\delta \gg 1$, i.e., $\left(\bar{\omega}_{c}-\omega_{\mathrm{M}}\right) / \omega_{\mathrm{M}} \gg$ $1 /\left(2 Q_{\mathrm{M}}\right)$, the shift $\Delta \omega_{c}$ is $Q$ independent.

It is tempting to expand on the single-mode approximation above by adding the contributions of many modes. This mode-sum approach is fundamentally flawed because the real part is infinite [54]. A linear divergence arises from the inclusion of the electron self-field contribution to the cavity radiation rather than only the field reflected from the walls. A calculation that explicitly removes the electron self-field from the cavity standing wave, i.e., "renormalizes" the field, yields a finite result and is the subject of the next section.

\section{Renormalized calculation of cyclotron-cavity coupling}

While it is possible to tackle the full cylindrical cavity directly, removing the electron self-field from such a calculation is difficult. It simplifies when first analyzing the interaction of the electron with two parallel plates then adding the contribution of interactions with the cylindrical wall. The calculation is quite involved. It is presented for a centered particle $(z, \rho=0)$ in Ref. [54]. We extend the calculation to any position in the cavity. This extension is required for two reasons: our electrostatic quadrupole suspends the electron slightly offset axially from the cavity mode center (see Sec. VD), and the electron's axial motion modulates the electron-cavity coupling when measuring the cyclotron damping rate (see Sec. V C 5). The derivation for arbitrary position is nearly identical to that in Ref. [54] except we keep the terms that vanish for $z, \rho=0$. Rather than repeat the lengthy calculation, we state the results and discuss some important characteristics.

For two parallel conducting plates, i.e., the cylindrical cavity with $\rho_{0} \rightarrow \infty$, the boundary conditions may be satisfied with a series of image charges. Renormalizing this sum is trivial-simply omit the electron's contribution and leave that of the image charges, a result we describe below and call $\Sigma_{P}$. Proceeding to the calculation of the full cylindrical cavity and omitting the contribution from the endcaps leaves only the correction from the cylindrical wall, a result we call $\Sigma_{S}$. The final result is the sum of the contributions from the endcaps and the wall.

At a cavity-shifted cyclotron frequency $\omega$, the frequency shift $\Delta \omega_{c}=\omega-\bar{\omega}_{c}$ and damping rate $\gamma_{c}$ is given in terms of these two contributions $\left(\Sigma_{P}, \Sigma_{S}\right)$, the free space damping rate $\gamma_{c 0}$, and a quality factor $Q$ for all modes:

$$
\begin{aligned}
\Delta \omega_{c}-\frac{i}{2} \gamma_{c}= & -\frac{i}{2} \gamma_{c 0}+\omega\left\{\Sigma_{S}\left[\left(1+\frac{i}{2 Q}\right) \omega, z, \rho\right]\right. \\
& \left.+\Sigma_{P}\left[\left(1+\frac{i}{2 Q}\right) \omega, z\right]\right\} .
\end{aligned}
$$

The correction to $g / 2$ [Eq. (10)] is equal to the relative shift of the cyclotron frequency:

$$
\frac{\Delta g_{\mathrm{cav}}}{2}=\frac{\Delta \omega_{c}}{\omega}
$$

We use this formula in a slightly modified form to calculate the cavity shifts of the cyclotron frequency (see the discussion in the next subsection).

The renormalized calculation begins by modeling the effect of the cavity on the electron as an electric field $\mathbf{E}^{\prime}(\mathbf{r})$ arising from image charges in the walls. It modifies the transverse equation of motion to read

$$
\dot{\mathbf{v}}-\boldsymbol{\omega}_{c} \times \mathbf{v}+\frac{e}{m} \nabla V(\mathbf{r})+\frac{1}{2} \gamma_{c 0} \mathbf{v}=\frac{e}{m} \mathbf{E}^{\prime}(\mathbf{r}) .
$$

The longitudinal part of $\mathbf{E}^{\prime}(\mathbf{r})$ gives a negligible correction to the trapping potential $V(\mathbf{r})$ [54], but the transverse part generates the anticipated effects. Using the radiation gauge, $\nabla \cdot \mathbf{A}=0$, the electric field may be written as the time derivative of the vector potential. This vector potential satisfies the wave equation with a transverse current source and thus may be written as the convolution of that source-the moving electron-and a Green's function subject to the appropriate boundary conditions, see, e.g., [34], Secs.6.3-6.4. Combining the two transverse velocity components as $v=v_{x}-i v_{y}=$ $v_{0} e^{-i \omega t}$, one can then write Eq. (42) as Eq. (40), where $\Sigma_{P}$ and $\Sigma_{S}$ are proportional to Fourier transforms of the part of the Green's function that arises due to the presence of the cavity walls. Note that $\Sigma_{P}$ and $\Sigma_{S}$ are in general complex, with the real portion corresponding to a frequency shift and the imaginary portion to a modified damping rate.

As mentioned above, the method of images gives the parallel-plate contribution to the cyclotron frequency shift and damping rate. As a function of axial position $z$ and cyclotron frequency $\omega$, it is

$$
\begin{aligned}
\Sigma_{P}(\omega, z)= & -r_{0}\left[2 \sum_{j=1}^{\infty} F\left(4 j z_{0}\right)-\sum_{j=1}^{\infty} F\left[2(2 j-1) z_{0}+2 z\right]\right. \\
& \left.-\sum_{j=1}^{\infty} F\left[2(2 j-1) z_{0}-2 z\right]\right]
\end{aligned}
$$

where $r_{0}=e^{2} /\left(4 \pi \epsilon_{0} m c^{2}\right)$ is the classical electron radius and $F(z)$ is the Fourier transform of the aforementioned Green's function a distance $|z|$ from an electron or image charge:

$$
F(z)=\frac{1}{|z|}\left[e^{i \omega|z| / c}\left(1+\frac{i c}{\omega|z|}-\frac{c^{2}}{\omega^{2} z^{2}}\right)+\frac{c^{2}}{\omega^{2} z^{2}}\right] .
$$

The $j=0$ term has been removed from the first sum; this exclusion of the electron self-field is the explicit renormalization required to avoid an infinite result.

$\Sigma_{P}$ depends on an axial offset but not a radial one because of the transverse symmetry of two parallel plates. Its imaginary part-cyclotron damping - has a sawtooth form with sharp teeth where the frequency corresponds to an integral number of half-wavelengths between the two endcaps (only the odd integers for $z=0$ since the even integers have a node there). The real part-cyclotron frequency shifts-shows peaks at similar intervals. The nearest such frequency corresponds to eight half-wavelengths at $154.5 \mathrm{GHz}$, far enough away that the parallel-plate contribution is smooth in our experimental region of interest. 
The contribution from the cylindrical wall is

$$
\begin{aligned}
\Sigma_{S}(\omega, z, \rho)= & -\frac{r_{0}}{z_{0}} \sum_{p=1}^{\infty} \sin ^{2}\left[\frac{p \pi}{2}\left(\frac{z}{z_{0}}+1\right)\right] \sum_{m=0}^{\infty}[1+\operatorname{sgn}(m)]\left[\frac{K_{m}^{\prime}\left(\mu_{p} \rho_{0}\right)}{I_{m}^{\prime}\left(\mu_{p} \rho_{0}\right)} R_{I}\left(m ; \mu_{p} \rho\right)\right. \\
& \left.+\left(\frac{p \pi c}{2 \omega z_{0}}\right)^{2}\left(\frac{K_{m}\left(\mu_{p} \rho_{0}\right)}{I_{m}\left(\mu_{p} \rho_{0}\right)} R_{I}\left(m ; \mu_{p} \rho\right)-\frac{K_{m}\left(\frac{p \pi \rho_{0}}{2 z_{0}}\right)}{I_{m}\left(\frac{p \pi \rho_{0}}{2 z_{0}}\right)} R_{I}\left(m ; \frac{p \pi \rho}{2 z_{0}}\right)\right)\right]
\end{aligned}
$$

with

$$
\begin{gathered}
\mu_{p}=\sqrt{\left(\frac{p \pi}{2 z_{0}}\right)^{2}-\left(\frac{\omega}{c}\right)^{2}}, \\
R_{I}(m ; x)=\frac{m^{2}}{x^{2}} I_{m}(x)^{2}+I_{m}^{\prime}(x)^{2}, \\
\operatorname{sgn}(m)=\left\{\begin{array}{lll}
-1 & \text { for } & m<0 \\
0 & \text { for } & m=0 . \\
1 & \text { for } & m>0
\end{array}\right.
\end{gathered}
$$

The sums include modified Bessel functions of the first and second kinds,

$$
\begin{gathered}
I_{v}(x)=i^{-v} J_{v}(i x) \\
K_{v}(x)=(\pi / 2)\left\{\left[I_{-v}(x)-I_{v}(x)\right] / \sin (v \pi)\right\}
\end{gathered}
$$

as well as their derivatives. The $K_{m}^{\prime}\left(\mu_{p} \rho_{0}\right) / I_{m}^{\prime}\left(\mu_{p} \rho_{0}\right)$ term comes from the boundary conditions of the TE modes, while the $K_{m}\left(\mu_{p} \rho_{0}\right) / I_{m}\left(\mu_{p} \rho_{0}\right)$ term comes from the TM modes. For $\rho \rightarrow 0$, the $R_{I}$ functions all go to zero except when $m=1$, when it goes to $1 / 2$. For $z \rightarrow 0$, only the odd- $p$ terms survive. Combined, these limits reproduce Eq. 4.28 of Ref. [54].

The frequency shifts and damping from individual modes can be seen by looking at the Bessel functions in the denominators. For a given $p$, an increasing $\omega$ will eventually cross a threshold at which $\mu_{p}$ becomes zero and then imaginary. At that point, we may use the definition of $I_{m}(x)$ to substitute

$$
I_{m}\left(\mu_{p} \rho_{0}\right)=i^{-m} J_{m}\left(\tilde{\mu}_{p} \rho_{0}\right),
$$

where $\tilde{\mu}_{p}$ is the now-real quantity $i \mu_{p}$. Since $J_{m}(x)$ and $J_{m}^{\prime}(x)$ have a number of zeros, after $\omega$ exceeds the $p$ th threshold the sum has poles that may be approximated as

$$
\Sigma_{S}(\omega, z, \rho) \approx \frac{\lambda_{m n p}^{2}}{\omega^{2}-\omega_{m n p}^{2}} .
$$

For TE modes, the poles occur when $J_{m}^{\prime}\left(\tilde{\mu}_{p} \rho_{0}\right)$ has a zero; for TM modes, when $J_{m}^{\prime}\left(\tilde{\mu}_{p} \rho_{0}\right)$ has a zero. That is, there are poles when $\omega=\omega_{m n p}$ of Eq. (13). Expanding the Bessel functions about their zeros yields mode coupling strengths

$$
\begin{aligned}
\mathrm{TE}: \lambda_{m n p}^{2}= & \frac{2 r_{0} c^{2}}{z_{0} \rho_{0}^{2}} \frac{-[1+\operatorname{sgn}(m)]}{J_{m}^{\prime \prime}\left(x_{m n}^{\prime}\right) J_{m}\left(x_{m n}^{\prime}\right)} \\
& \times \sin ^{2}\left[\frac{p \pi}{2}\left(\frac{z}{z_{0}}+1\right)\right] R_{J}\left(m ; x_{m n}^{\prime} \frac{\rho}{\rho_{0}}\right) \\
\mathrm{TM}: \lambda_{m n p}^{2}= & \frac{2 r_{0} c^{2}}{z_{0} \rho_{0}^{2}} \frac{1+\operatorname{sgn}(m)}{J_{m}^{\prime}\left(x_{m n}\right)^{2}}\left(\frac{p \pi}{2 z_{0}} \frac{c}{\omega_{m n p}}\right)^{2} \\
& \times \sin ^{2}\left[\frac{p \pi}{2}\left(\frac{z}{z_{0}}+1\right)\right] R_{J}\left(m ; x_{m n} \frac{\rho}{\rho_{0}}\right),
\end{aligned}
$$

where $x_{m n}$ and $x_{m n}^{\prime}$ are the previously mentioned zeros of the Bessel functions and their derivatives. The entire radial dependence of the coupling is contained in the $R_{J}$ function, defined by

$$
R_{J}(m ; x)=\frac{m^{2}}{x^{2}} J_{m}(x)^{2}-J_{m}^{\prime}(x)^{2} .
$$

For zero radius, $R_{J}$ equals $1 / 2$ if $m=1$ and zero otherwise.

Given the above, we can see that the summation indices $p$ and $m$ in Eq. (45) correspond directly to those in the mode indices $m n p$, and the addition of the $m, p$ th term of the sums adds the contributions from all modes of that $m$ and $p$. The threshold above which $\mu_{p}$ is imaginary corresponds to the frequency whose half-wavelength fits between the endcaps $p$ times. The single-mode approximation of Eq. (52) shows the interaction between the electron and a single mode is that of two weakly coupled oscillators.

\section{Using the renormalized calculation}

The combination of $\Sigma_{P}$ and $\Sigma_{S}$ in Eq. (40) is the result of the renormalized calculation. There, we have included cavity dissipation in the from of a mode $Q$ with the replacement $\omega \rightarrow[1+i /(2 Q)] \omega$. It is possible to include different quality factors for the TE and TM mode classes by using $Q_{\mathrm{TE}}$ in the denominator functions $I_{m}^{\prime}\left(\mu_{p} \rho_{0}\right)$ and $Q_{\mathrm{TM}}$ everywhere else [54]. It is not possible to include a $Q$ for each mode separately.

The strength of the renormalized calculation is its removal of the electron self-energy. It has an important drawback in that the entire calculation has only four input parameters: $\rho_{0}$, $z_{0}, Q_{\mathrm{TM}}$, and $Q_{\mathrm{TE}}$. It does not allow the input of arbitrary mode frequencies and $Q$ s. If the dominant mode couplings are to one TE and one TM mode, then the two mode frequencies and $Q$ s can determine the four input parameters. The addition of a third mode, however, overconstrains the problem; unless the three modes happen to have frequencies that correspond to those of an ideal cavity and two happen to share $Q$ s, the renormalized calculation will give an incorrect result.

In this measurement, three modes have the largest influence on the electron-cavity coupling: $\mathrm{TE}_{127}, \mathrm{TM}_{143}$, and $\mathrm{TE}_{136}$. Because we can identify the terms in Eq. (45) that correspond to a given mode, we modify the renormalized calculation to better approximate our observed mode structure. Since the electron is close to (but not precisely in) the mode center, the two modes with antinodes at the center (odd $p$ ) dominate the coupling, and we set the four input parameters of the renormalized calculation with the frequencies and $Q$ s of $\mathrm{TE}_{127}$ and $\mathrm{TM}_{143}$. These input parameters give an ideal frequency and $Q$ for $\mathrm{TE}_{136}$, which we correct to the observed values by subtracting the term in Eq. (45) that includes the contribution from $\mathrm{TE}_{136},-\left(2 r_{0} / z_{0}\right) \sin ^{2}\left[3 \pi\left(z / z_{0}+1\right)\right]$ 
$\left[K_{1}^{\prime}\left(\mu_{6} \rho_{0}\right) / I_{1}^{\prime}\left(\mu_{6} \rho_{0}\right)\right] R_{I}\left(1 ; \mu_{6} \rho\right)$, and adding it back with the observed values. Although this moves all modes with $\mathrm{TE}_{1 n 6}$ the next-nearest ones are far away: $\omega_{\mathrm{TE} 126} /(2 \pi) \approx 129 \mathrm{GHz}$ and $\omega_{\mathrm{TE} 146} /(2 \pi) \approx 169 \mathrm{GHz}$, while $\omega_{\mathrm{TE} 136} /(2 \pi) \approx 146 \mathrm{GHz}$. If this were a concern, we could do a similar subtraction with the single-mode approximation of Eq. (52), but at the cost of artifacts of uncanceled higher-order terms.

\section{Coupling modulated by axial motion}

When the SEO is running, the axial motion through the cavity mode field will modulate the coupling at $\omega_{z}$. This motion is present, for example, during cyclotron or spin state-detection; it is not present during cyclotron or anomaly excitation, the relevant period for any systematic shift in $g / 2$. Since measurements of the cyclotron damping rate involve continuous detection of the cyclotron state waiting for a decay, we must account for this modulation and any amplitude dependence of the damping rate when using measurements of $\gamma_{c}$ to determine the input parameters to the $g / 2$ cavity corrections. This accounting is a nontrivial task, which is intractable for the full renormalized calculation but doable for the single-mode coupling of Eq. (52) provided that the axial amplitude $A$ is much lower than a quarter-wavelength of the mode's axial standing wave $\left(A \ll z_{0} / p\right)$, a condition met for typical $A$.

All of the $z$ dependences in the mode-coupling parameters $\lambda_{m n p}$ of Eq. (53) come in a single sine function:

$$
\lambda_{\text {mnp }}=\sin \left[\frac{p \pi}{2}\left(\frac{z}{z_{0}}+1\right)\right] \tilde{\lambda}_{\mathrm{M}},
$$

where we define $\tilde{\lambda}_{M}$ to be the non- $z$-dependent part of the coupling. For an electron offset $z$ from the center of the modes and oscillating at frequency $\omega_{z}$ with amplitude $A \ll z_{0} / p$, we may expand the mode axial dependence in terms of axial harmonics,

$\sin \left[\frac{p \pi}{2}\left(\frac{z+A \cos \left(\omega_{z} t\right)}{z_{0}}+1\right)\right]=\sum_{j=0}^{\infty} f_{j}(z, A) \cos \left(j \omega_{z} t\right)$,

where the $f_{j}(z, A)$ are functions of the axial offset and amplitude. The first three are

$$
\begin{gathered}
f_{0}(z, A)=\sin \left[\frac{p \pi}{2}\left(\frac{z}{z_{0}}+1\right)\right]\left[1-\left(\frac{p \pi A}{4 z_{0}}\right)^{2}+O\left(A^{4}\right)\right] \\
f_{1}(z, A)=\cos \left[\frac{p \pi}{2}\left(\frac{z}{z_{0}}+1\right)\right]\left[\frac{p \pi A}{2 z_{0}}-O\left(A^{3}\right)\right], \\
f_{2}(z, A)=\sin \left[\frac{p \pi}{2}\left(\frac{z}{z_{0}}+1\right)\right]\left[-\left(\frac{p \pi A}{4 z_{0}}\right)^{2}+O\left(A^{4}\right)\right] .
\end{gathered}
$$

Including this expansion of the axial oscillation in the transverse equation of motion ( [12], Appendix A) yields an amplitude dependence to the single-mode coupling strength as well as a series of axial harmonics to the mode frequency, $\omega_{\mathrm{M}}$ :

$$
\begin{aligned}
\Delta \omega_{c}-i \frac{\gamma_{c}}{2}= & \frac{\tilde{\lambda}_{\mathrm{M}}^{2} \omega}{2} \sum_{j=0}^{\infty} f_{j}(z, A)^{2}\left[\frac{1}{\omega^{2}-\left(\omega_{\mathrm{M}}-j \omega_{z}\right)^{2}}\right. \\
& \left.+\frac{1}{\omega^{2}-\left(\omega_{\mathrm{M}}+j \omega_{z}\right)^{2}}\right]
\end{aligned}
$$

As before, we may include a damping width by substituting $\omega \rightarrow[1+i /(2 Q)] \omega$ in the two fractions within the brackets. Note that taking the $A \rightarrow 0$ limit recovers the usual singlemode coupling of Eq. (52).

\section{Single-electron mode detection}

Using the above descriptions of electron-cavity coupling, we measure the cyclotron damping rate as a function of cyclotron frequency and of position in the trap to determine the location and $Q$ of the three closest coupled modes and to characterize the alignment of the electrostatic and mode centers. Since we are able to perform a QND measurement on the cyclotron state, measuring the cyclotron damping rate simply consists of making many (typically hundreds of) jumps and fitting the distribution of jump lengths to a decaying exponential with time-constant $\gamma_{c}^{-1}$, as in Fig. 11(a). Because the axial motion is self-excited during detection, we must measure the damping rate as a function of amplitude. The amplitude dependence goes as even powers of $A$ [Eqs. (57) and (58)], and since the amplitude is much less than a quarter-wavelength for the relevant modes, terms of higher order in $A$ get progressively smaller, allowing approximation as a quadratic function,

$$
\gamma(A)=\gamma_{0}+\gamma_{2} A^{2} .
$$

We measure the axial amplitude with the driven cyclotron lineshape of Eq. (30), where the driven axial motion in the
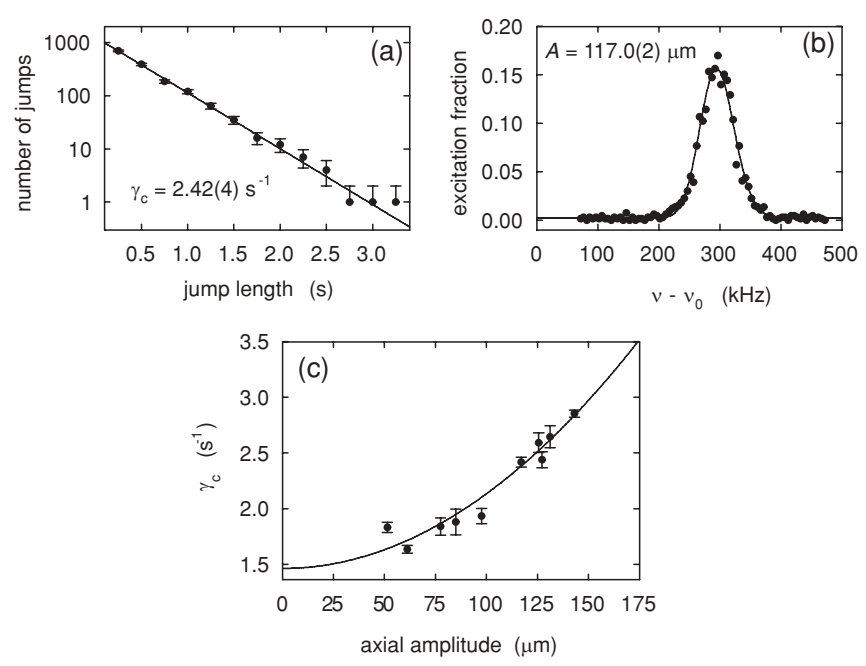

FIG. 11. Measurement of the cyclotron damping rate at 146.70 $\mathrm{GHz}$, near the upper sideband of $\mathrm{TE}_{136}$. The cyclotron damping rate as a function of axial amplitude (c) extrapolates to the desired lifetime. Each point in (c) consists of a damping rate measured from a fit to a histogram of cyclotron jump lengths (a) as well as an axial amplitude measured from a driven cyclotron line (b). 
magnetic bottle causes the electron to see a higher average magnetic field, resulting in a cyclotron frequency shift as in Fig. 11(b). Figure 11(c) shows an example of the measured damping rate as a function of amplitude close to the upper axial sideband of $\mathrm{TE}_{136}\left(v_{\mathrm{TE} 136}+v_{z}\right)$. It displays a large amplitude dependence in $\gamma_{c}$ because of the proximity of $\bar{\nu}_{c}$ and the sideband, which becomes more prominent as larger axial oscillations increase the modulation of the mode coupling.

After repeating such measurements at many cyclotron frequencies, we amass two sets of data, the zero-amplitude cyclotron decay rates $\gamma_{0}$ and the quadratic amplitude-dependence coefficients $\gamma_{2}$, as functions of $\bar{v}_{c}$. From these data, we extract the frequencies of the three nearest coupled modes: $\mathrm{TE}_{127}$,
$\mathrm{TE}_{136}$, and $\mathrm{TM}_{143}$. Since the lifetimes are heavily $Q$ dependent, we must include three $Q$ s, bringing the total number of fit parameters to six.

The renormalized calculation provides the model for fitting the zero-amplitude cyclotron decay rates. As described above, we use its four free parameters to determine the frequencies and $Q$ s of $\mathrm{TE}_{127}$ and $\mathrm{TM}_{143}$ and add the $\mathrm{TE}_{136}$ parameters by subtracting the $\mathrm{TE}_{1 n 6}$ term in the renormalized calculation for the calculated frequency and $Q$ and adding it back in with the frequency and $Q$ as fit parameters.

For the quadratic-amplitude-dependence data, we use the $A^{2}$ term in the single-mode coupling expansion of Eq. (58) to write

$$
\begin{aligned}
\gamma_{2}=-2 & \sum_{\mathrm{M}} \operatorname{Im}\left\{\frac { \tilde { \lambda } _ { \mathrm { M } } ^ { 2 } \omega } { 2 } \left[\sin ^{2}\left[\frac{p \pi}{2}\left(\frac{z}{z_{0}}+1\right)\right]\left(\frac{p \pi}{4 z_{0}}\right)^{2} \frac{-4}{\omega^{2}+i \frac{\omega \omega_{\mathrm{M}}}{Q_{\mathrm{M}}}-\omega_{\mathrm{M}}^{2}}+\cos ^{2}\left[\frac{p \pi}{2}\left(\frac{z}{z_{0}}+1\right)\right]\left(\frac{p \pi}{2 z_{0}}\right)^{2}\right.\right. \\
& \left.\left.\times\left(\frac{1}{\omega^{2}+i \frac{\omega\left(\omega_{\mathrm{M}}-\omega_{z}\right)}{Q_{\mathrm{M}}}-\left(\omega_{\mathrm{M}}-\omega_{z}\right)^{2}}+\frac{1}{\omega^{2}+i \frac{\omega\left(\omega_{\mathrm{M}}+\omega_{z}\right)}{Q_{\mathrm{M}}}-\left(\omega_{\mathrm{M}}+\omega_{z}\right)^{2}}\right)\right]\right\}
\end{aligned}
$$

where the sum is over the three modes of interest.

We form a $\chi_{\gamma_{0}}^{2}$ using the zero-amplitude data and the renormalized calculation and a $\chi_{\gamma_{2}}^{2}$ using the quadraticamplitude-dependence data and Eq. (60). Since the electron is close to centered axially (we discuss the offset below and include the measured offset in the fits), the zero-amplitude data are more sensitive to the two modes with central antinodes $\left(\mathrm{TE}_{127}\right.$ and $\left.\mathrm{TM}_{143}\right)$ and the amplitude-dependence data to the sidebands of $\mathrm{TE}_{136}$, which has a central node. The fit consists of minimizing the two $\chi^{2} \mathrm{~s}$; though the proper weighting of the two is not clear a priori, it makes little difference to the results.

Figures 12(b) and 12(c) display the lifetime data and fits, and Table II lists the results and compares them to those from the parametric mode maps. The two independent methods should agree but do not. When calculating the cavity shifts, we assign uncertainties large enough to include both results for the mode frequencies (see Table III). For the mode $Q$ s, to which the cavity shifts are largely insensitive, we use the

TABLE III. Parameters used in calculating the cavity shifts. For comparison, we include earlier estimates from the 2006 measurement, which used the same trap cavity.

\begin{tabular}{lccc}
\hline \hline & & $\begin{array}{c}2006 \\
\text { measurement [1] }\end{array}$ & $\begin{array}{c}\text { this } \\
\text { measurement }\end{array}$ \\
\hline $\mathrm{TE}_{127}$ & $v_{c}(\mathrm{GHz})$ & $146.350(200)$ & $146.309(27)$ \\
& $Q$ & $>500$ & $4900(300)$ \\
$\mathrm{TE}_{136}$ & $v_{c}(\mathrm{GHz})$ & - & $146.428(15)$ \\
& $Q$ & - & $4800(200)$ \\
$\mathrm{TM}_{143}$ & $v_{c}(\mathrm{GHz})$ & $151.900(200)$ & $151.832(37)$ \\
& $Q$ & $>500$ & $1270(70)$ \\
electrostatic & $\mathrm{z}(\mu \mathrm{m})$ & 0 & $165(4)$ \\
offset & $\rho(\mu \mathrm{m})$ & 0 & $<10$ \\
\hline \hline
\end{tabular}

results from the lifetime fits because of the strong-coupling ambiguity in the parametric mode map values.

\section{Electron position in the cavity}

Knowledge of the electron position relative to the cavity modes is important for calculating the electron-mode coupling and thus the cyclotron frequency shift. We determine the axial misalignment by measuring the cyclotron damping rate as a function of $z$, using antisymmetric endcap potentials to move the electron along the trap axis [6]. Figure 13 plots such a measurement with the cyclotron frequency tuned midway between $\mathrm{TE}_{136}$ and its upper sideband-close enough to $\mathrm{TE}_{136}$ to give a large $z$ dependence but far enough detuned that the cyclotron lifetime is long enough to see single excitations. Since the mode couplings are even functions of $z$, the damping rate should go as $z^{2}$ with an extremum at $z=0$ for the modes. Figure 13 shows this extremum, which fits to a misalignment of $165(4) \mu \mathrm{m}$. In addition, since the extremum is a minimum, the nearest coupled mode must have a node at the mode center (even $p$ ), demonstrating that the $\mathrm{TE}_{127}$ and $\mathrm{TE}_{136}$ identifications in Fig. 10 are correct.

The cause of this offset is not known, though the consistent presence of $\mathrm{TE}_{136}$ in the parametric mode maps suggests that it is stable. Measurement of a third trap "center," the minimum of the magnetic bottle, agrees with the electrostatic center. Attempts to model the observed offset as asymmetric spacing between electrodes does not yield a convincing explanation and more exotic trap deformations such as a tilted endcap or a compensation electrode that protrudes slightly into the cavity are difficult to model. Because both the parametric mode maps and Fig. 13 indicate an offset, we include it when calculating the cavity shifts. We build our confidence in this procedure by measuring $g / 2$ at four cyclotron frequencies with different cavity shifts and showing the agreement between the predicted and measured shifts. 
We estimate the radial alignment of a single electron by tuning its cyclotron frequency into resonance with three modes that have nodes at the radial center, i.e., have $m \neq 1$, and comparing the measured cyclotron damping rate to that predicted by the renormalized model with $\rho=0$. Since the $m \neq 1$ modes do not couple to a radially centered electron, a measured cyclotron damping rate that is faster than the calculated damping rate could indicate a radial misalignment. For the cases where we observe such a discrepancy, we use the full, $\rho$-dependent renormalized calculation to estimate the range of radial offsets that could explain the observed damping rates (all calculations include the axial offset of the previous paragraphs). In each of the three cases $\left(\mathrm{TE}_{035}, \mathrm{TM}_{027}, \mathrm{TE}_{043}\right)$, we measure damping rates close to that predicted for $\rho=0$, and we set the limit $\rho<10 \mu \mathrm{m}$.

\section{E. Cavity-shift results}

We calculate the cavity shifts and uncertainties from the mode parameters and their uncertainties (Table III) via the renormalized calculation. The cavity shifts are independent of mode $Q$ for cyclotron frequencies with relative detunings $\left(\bar{\omega}_{c}-\omega_{\mathrm{M}}\right) / \omega_{\mathrm{M}} \gg 1 /\left(2 Q_{\mathrm{M}}\right)$. Because all our $g / 2$ measure-

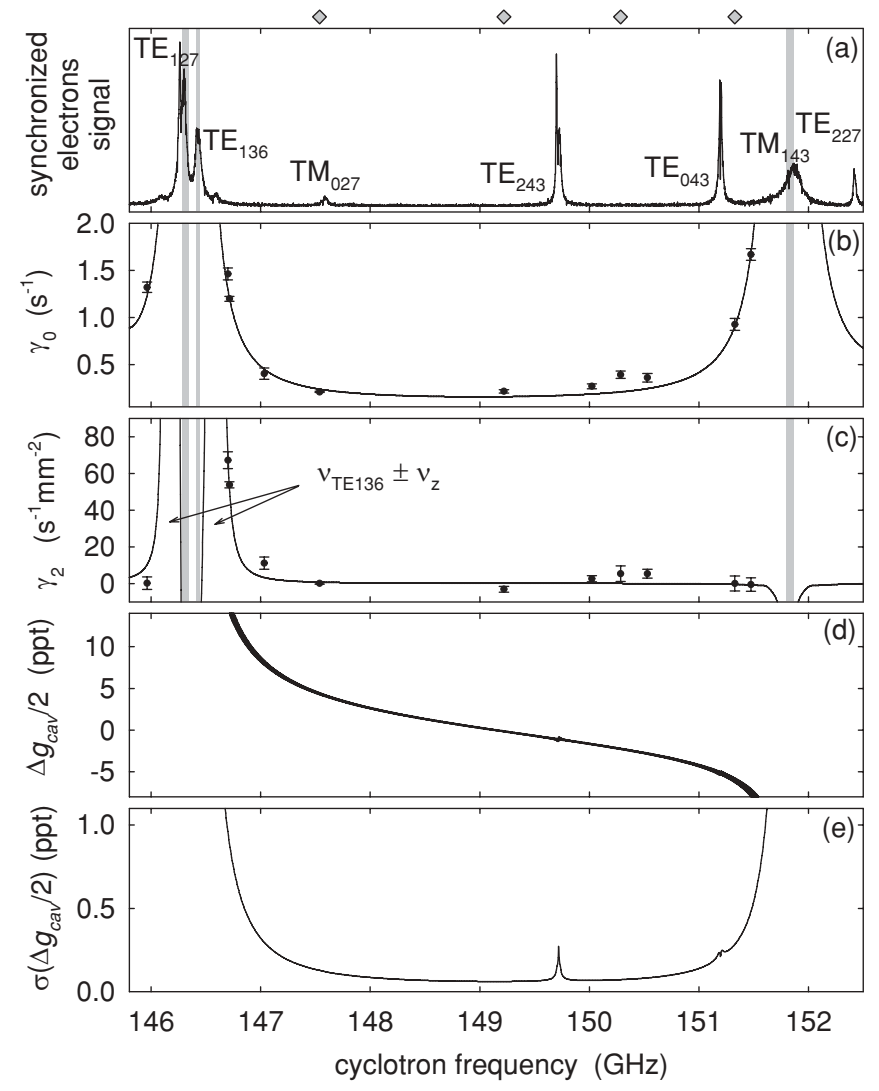

FIG. 12. Cavity-shift results come from synchronized electrons (a) and from direct measurements with one electron of $\gamma_{c}$ (b) and its dependence on axial amplitude (c). Together, they provide uncertainties in the frequencies of coupled cavity modes (gray) that translate into an uncertainty band of cavity shifts $\Delta g_{\text {cav }} / 2$ (d) whose half-width, i.e., the cavity-shift uncertainty, is plotted in (e). The diamonds at the top indicate the cyclotron frequencies of the four $g / 2$ measurements. ments meet this requirement easily, the cavity shift uncertainty comes only from the errors in mode frequencies. Because of the axial offset, mode $\mathrm{TE}_{136}$ affects the shifts, and we calculate with this mode at its observed frequency using the mode-moving technique described in Sec. VC4. Because fits to the parametric mode maps and to the single-electron lifetime data yield slightly different frequencies for the three nearest coupled modes (see Table II), we assign uncertainties large enough to include both. The trap-radius limit only has a significant effect near two modes, $\mathrm{TE}_{243}$ and $\mathrm{TE}_{043}$, and we again use the mode-moving technique to place them at their observed frequencies though it makes little difference to our result because none of our four measurements of $g / 2$ were resonant with either mode. Because it appears in the parametric mode map, we include $\mathrm{TM}_{027}$ in the calculation, although it does not change the result noticeably.

Figures 12(d) and 12(e) display the results of this analysis, and Table $\mathrm{V}$ shows the calculated cavity shifts for our four measurements of $g / 2$. The shifts span over $10 \mathrm{ppt}$ with uncertainties around 100 times smaller than that range. The lowest uncertainties are below a part in $10^{13}$, over six times smaller than in our 2006 measurement [1] and low enough that this systematic uncertainty is no longer a dominant error in the measurement of $g / 2$.

\section{POWER SHIFTS}

We expect neither $\bar{v}_{a}$ nor $\bar{f}_{c}$ to shift with cyclotron or anomaly drive power, but previous measurements of the electron $g / 2$ at the UW showed unexplained systematic shifts of the cyclotron frequency with both drive powers [4,30]. The origin of these power shifts in the UW measurements remains unknown [30], and extrapolation to zero cyclotron power involved correcting shifts of several ppt in $g / 2$ [4,30]. Estimates comparing our drive power to that used in the UW measurements suggest that our narrower lines and singlequantum cyclotron technique require drive strengths low enough that the power shifts are negligible. We have yet to see a power shift in our apparatus, though experimental searches are time-consuming and the statistical uncertainty in the current search is comparable to our final uncertainty in $g / 2$.

\section{A. Anomaly power shift estimate}

The UW experiment showed anomaly power shifts of several $\mathrm{ppb}$ in the anomaly frequency [30]. An off-resonant

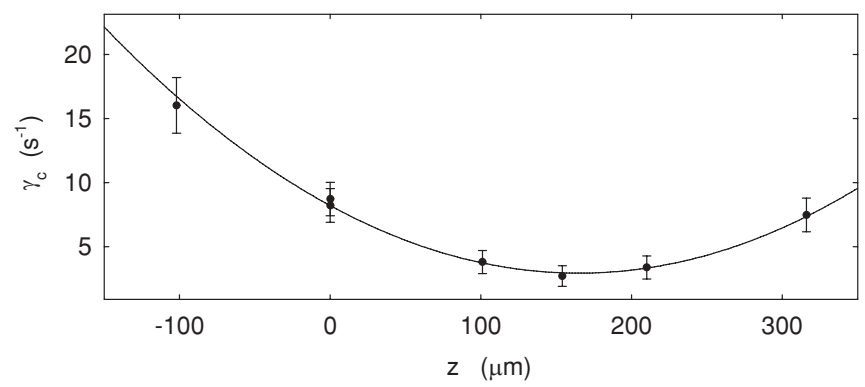

FIG. 13. Measurement of the offset between the electrostatic and mode centers. Here, $z$ refers to the position relative to the electrostatic center, and the minimum in the cyclotron damping rate corresponds to the mode center. 
anomaly drive during cyclotron excitation shifted the cyclotron line by a similar amount, and the two shifts canceled in the frequency-ratio calculation of $g / 2$ [4]. The origin of these shifts is unknown, although experiments with a variablestrength magnetic bottle showed that they increase with the magnitude of the bottle strength, independent of its sign [30].

Direct comparisons between the anomaly power used in the UW experiment and that used here are difficult because the experiments use different anomaly excitation techniques. The UW excitations were primarily driven with counterflowing current loops in split compensation electrodes, while we drive the electron axially through the $z \rho \hat{\rho}$ gradient of the magnetic bottle. Unlike the current-loop excitation technique, our axial-excitation technique provides a clear mechanism for an anomaly power shift by increasing the average axial amplitude and, therefore, the average magnetic field seen by the electron (because of the $z^{2} \hat{\mathbf{z}}$ part of the magnetic bottle). We estimate this shift below and expect it to be both smaller than our current precision and canceled in the calculation of $g / 2$ by a similar cyclotron shift from a detuned anomaly drive during cyclotron excitation.

For a driven axial amplitude, $z_{a}$, the frequency shift from the motion through the magnetic bottle is

$$
\frac{\Delta \omega_{a}}{\omega_{a}}=\frac{\Delta \omega_{c}}{\omega_{c}}=\frac{B_{2}}{B} \frac{z_{a}^{2}}{2} .
$$

Were this shift to approach the linewidth, we would be forced to use the driven lineshape of Eq. (30) when extracting frequencies. In order to calculate the expected size of the power shift, we must estimate $z_{a}$; only amplitudes over $800 \mathrm{~nm}$ will produce shifts at the $0.1 \mathrm{ppb}$ level in frequency. We estimate $z_{a}$ using two methods: the observed anomaly transition rate and a calibration of the drive voltage. The estimates give similar amplitudes, and neither predicts anomaly power shifts at our precision.

From Eq. (35), we see that the anomaly transition rate goes as the product of the Rabi frequency squared $\Omega_{a}^{2}$ times the lineshape function $\chi(\omega)$. The anomaly Rabi frequency goes as $z_{a}$ [51], and we can estimate $z_{a}$ from a typical peak excitation fraction, $P_{\mathrm{pk}}$, using

$$
\begin{aligned}
P_{\mathrm{pk}} & =\frac{\pi}{2} T \Omega_{a}^{2} \chi\left(\omega_{\mathrm{pk}}\right) \\
& =\frac{\pi}{2} T\left(\frac{g}{2} \frac{e \hbar}{2 m} B_{2} z_{a} \sqrt{\frac{2}{m \hbar\left(\bar{\omega}_{c}-\bar{\omega}_{m}\right)}}\right)^{2} \chi\left(\omega_{\mathrm{pk}}\right) .
\end{aligned}
$$

Because the lineshape function is normalized to unity, its value on-peak in the Lorentzian lineshape limit-the limit corresponding to the anomaly line-is inversely-proportional to the linewidth

$$
\chi\left(\omega_{\mathrm{pk}}\right)=\frac{2}{\pi}\left(\frac{2 \Delta \omega^{2}}{\gamma_{z}}+\gamma_{c}\right)^{-1} .
$$

For typical experimental parameters, we must drive to $z_{a} \approx$ $100 \mathrm{~nm}$ to achieve a $20 \%$ excitation fraction.
Alternately, we can estimate the driven amplitude based on the rf voltage on the bottom endcap. An endcap driven with amplitude $V_{a}$ excites the electron to an amplitude given by [29]

$$
z_{a}=\frac{c_{1} d^{2}}{2 z_{0}}\left[\left(\frac{\omega_{a}}{\omega_{z}}\right)^{2}-1\right]^{-1} \frac{V_{a}}{V_{R}}
$$

( $V_{R}$ is the ring electrode potential, and $c_{1}, d$, and $z_{0}$ are geometric factors equal to approximately $0.78 \mathrm{~mm}, 3.5 \mathrm{~mm}$, and $3.8 \mathrm{~mm}$ in our trap.)

We determine the drive amplitude $V_{a}$ by measuring the power output of our frequency synthesizer and using two methods to calibrate the attenuation in the drive line to the bottom endcap electrode. First, we look at shifts the anomaly drive induces in the axial frequency. The shifts arise from both a frequency-pulling from the off-resonant axial force and a Paul-trap shift from the change in effective trapping potential [51]. They amount to $\Delta \bar{v}_{z} /\left(\bar{v}_{z} V_{a}^{2}\right)=-37 \mathrm{ppm} V^{-2}$. By increasing the output power of the signal generator far above that used for anomaly excitation, we determine that the drive line attenuates the signal by $30 \mathrm{~dB}$. This agrees with the attenuation we measure in the drive line during room-temperature calibrations and seems reasonable given the $20 \mathrm{~dB}$ cold attenuator installed at the $1 \mathrm{~K}$ pot and some additional loss in the stainless steel semirigid coaxial cable. The highest anomaly power used for $g / 2$ data was $-16 \mathrm{dBV}$ at the synthesizer, which would attenuate to $V_{a}=5 \mathrm{mV}$ at the bottom endcap and drive the electron to $z_{a}=250 \mathrm{~nm}$.

Driven axial amplitudes around 100-250 nm should only shift the anomaly and cyclotron frequencies at the $1-10 \mathrm{ppt}$ level, which is far too small to affect the lineshapes (the error in $g / 2$ would be lower by 1000 if the cyclotron and anomaly shifts were uncorrelated, but should be even smaller because the shifts cancel in the frequency ratio).

\section{B. Cyclotron power shift estimate}

Unlike the anomaly power shifts, the cyclotron power shifts seen in the UW experiments did not cancel in $g / 2$ and added a $1.3 \mathrm{ppt}$ uncertainty to their 1987 result [4]. The shifts appeared as a resonant effect of unknown origin with a resonant drive shifting the cyclotron line several ppb but a detuned cyclotron drive with the same power not shifting the anomaly line. Investigations in a trap with a variable-strength magnetic bottle showed that the shift scaled with $B_{2}$ in magnitude and sign. In [30], the authors hypothesize that the shift could have originated in an excitation of the magnetron motion because a typical shift could have been explained by a $10 \%$ increase of the magnetron radius.

Our cyclotron excitation technique, injecting microwaves into the trap cavity, is similar to that used in the UW measurements, so we can compare our technique to theirs. Our lower temperature narrows the lines by a factor of 10 , requiring less power to drive transitions. The measured bottle-dependence suggests that our 10-times-stronger magnetic bottle could cancel the advantage of our narrower lines. The overall shifts should still be reduced because our single-quantum-jump spectroscopy only needs to excite to the $n=1$ state less than $20 \%$ of the time. At the UW, typical excitations sustained the electron at energies corresponding to $n \gtrsim 4[29,30]$. Naively, 


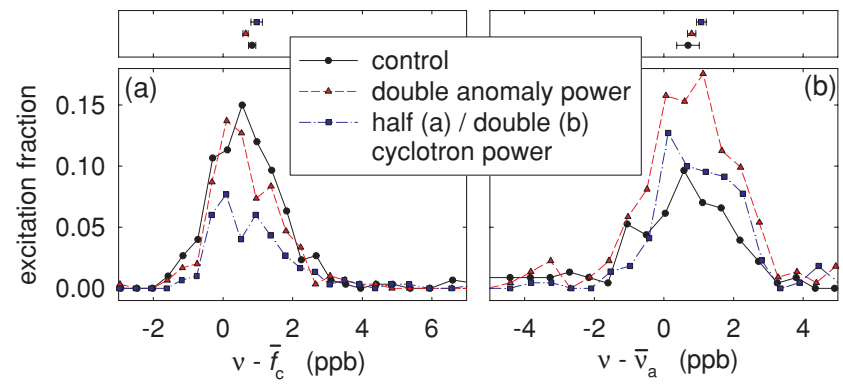

FIG. 14. (Color online) The lower plots show cyclotron (a) and anomaly (b) data taken in search of cyclotron and anomaly power shifts. The upper plots compare the weighted means of these lines.

exciting to an average energy of $n=4$ requires 20-times more power than an average energy of $n=0.2$, and this power reduction alone would reduce several-ppt shifts in $g / 2$ below our precision. The relativistic shifts between cyclotron levels suggest additional power in the UW drives because excitations above $n=1$ involve driving in the exponential tails of the higher states' resonances. In addition, if the power shift is indeed related to driving a magnetron-cyclotron sideband, our ten-times-higher magnetron frequency and 10-times-narrower cyclotron lines put the closest magnetron sideband, which was fewer than 10 linewidths away at the UW, 100 times farther from the cyclotron resonance.

\section{Experimental search for power shifts}

Although we do not expect any cyclotron or anomaly power shifts of $\bar{v}_{a}$ or $\bar{f}_{c}$, their existence in the UW measurements makes us proceed with caution and look for them anyway. We examine the shifts of each line individually to ensure that no systematic effects (even ones that cancel in $g / 2$ ) go unnoticed. We look for a cyclotron frequency shift by running three cyclotron scans: a control, one with double the detuned anomaly power, and one with half the cyclotron power (lower to avoid saturation). The scans are interleaved in the same way we interleave cyclotron and anomaly scans during $g / 2$ measurements, alternating single sweeps of each line and including edge-tracking to remove long-term drifts (see Sec. III). The resulting cyclotron lines are shown in Fig. 14(a). We calculate the cyclotron frequency of each line with the weighted-mean method (the offset from $\bar{f}_{c}$ cancels when subtracting for a frequency shift). Frequency differences between methods are summarized in Table IV.

To look for anomaly frequency shifts, we run three anomaly scans-a control, one with double the detuned cyclotron power, and one with double the anomaly power (the control power is low enough that we can double the power without saturating)—interleaved and normalized via edge tracking as

TABLE IV. Summary of power-shift searches.

\begin{tabular}{lr}
\hline \hline test & "shift" (ppb) \\
\hline $\bar{f}_{c}$ with double anomaly power & $-0.18(13)$ \\
$\bar{f}_{c}$ with half cyclotron power & $0.13(19)$ \\
$\bar{v}_{a}$ with double anomaly power & $0.11(35)$ \\
$\bar{v}_{a}$ with double cyclotron power & $0.38(35)$ \\
\hline \hline
\end{tabular}

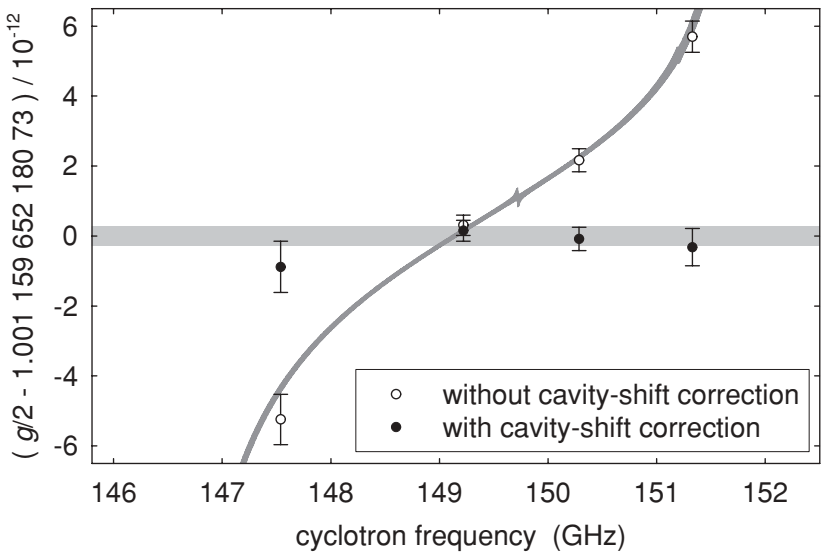

FIG. 15. Four measurements of $g / 2$ without (open) and with (filled) cavity-shift corrections. The light gray uncertainty band shows the average of the corrected data. The dark-gray band indicates the expected location of the uncorrected data given our result in Eq. (65) and including only the cavity-shift uncertainty.

before. The resulting anomaly lines are shown in Fig. 14(b), which includes the frequencies calculated by the weightedmean method. Table IV summarizes the differences.

The results in Table IV as consistent with zero. The largest "shift" is that of the anomaly frequency with cyclotron power-the only one of the four not seen at the UW. The data of Table IV suggest that any power shift will be $\lesssim 0.35$ ppb in frequency, which is consistent with the limits of our prior studies (detailed in Sec. 6.2 of Ref. [41]) and with our expectation of no shift at our current precision. The uncertainties are limited by our ability to resolve the lines in a timely manner. (The number of nights spent assembling the data in Fig. 14 exceeds half the number used to determine the $g / 2$ value.) The anomaly line in particular requires the time-consuming discrimination between $|0, \uparrow\rangle$ and $|1, \downarrow\rangle$ after each anomaly pulse, and any search for a systematic shift in the anomaly frequency multiplies the number of times this must occur. Because we estimate that no power shift should occur and our experimental searches are limited by our ability to resolve the lines, we apply neither a correction nor any additional uncertainty from power shifts.

\section{RESULTS AND APPLICATIONS}

\section{A. Most accurate determination of the dlectron $g$ value}

The result for the electron magnetic moment in Bohr magnetons,

$$
g / 2=1.00115965218073(28) \quad \text { [0.28ppt }],
$$

comes from the weighted average of the four measurements with uncorrelated and correlated uncertainties combined appropriately. The result has 2.7 and 15 times lower uncertainty than the 2006 and 1987 measurements and 2300 times lower uncertainty than has been achieved for the heavier $\mu$ lepton [5]. Table V summarizes the measured values, shifts, and uncertainties for the four separate measurements of $g / 2$. The uncertainties are lower for measurements with smaller cavity shifts and narrower linewidths, as might be expected. While significant cavity shifts exist (see Fig. 15), their uncertainties 
TABLE V. Measurements and shifts with uncertainties, multiplied by $10^{12}$. The cavity-shifted " $g / 2$ raw" and corrected " $g / 2$ " are offset from our result in Eq. (65).

\begin{tabular}{lrrrr}
\hline \hline$\overline{f_{c}}$ & $147.5 \mathrm{GHz}$ & $149.2 \mathrm{GHz}$ & $150.3 \mathrm{GHz}$ & $151.3 \mathrm{GHz}$ \\
\hline$g / 2$ raw & $-5.24(0.39)$ & $0.31(0.17)$ & $2.17(0.17)$ & $5.70(0.24)$ \\
Cav. shift & $4.36(0.13)$ & $-0.16(0.06)$ & $-2.25(0.07)$ & $-6.02(0.28)$ \\
Lineshape & & & & \\
correlated & $(0.24)$ & $(0.24)$ & $(0.24)$ & $(0.24)$ \\
uncorrelated & $(0.56)$ & $(0.00)$ & $(0.15)$ & $(0.30)$ \\
\hline$g / 2$ & $-0.88(0.73)$ & $0.15(0.30)$ & $-0.08(0.34)$ & $-0.32(0.53)$ \\
\hline \hline
\end{tabular}

no longer dominate. We no longer quote uncertainties for variations of the power of the $\bar{v}_{a}$ and $\bar{f}_{c}$ drives; although power-shifts appeared in the 1987 measurement [4,30], our narrower lines and single-quantum cyclotron technique require much lower drive powers and we estimate-and check experimentally - that they are no longer important.

\section{Relation of 2006 and 2008 measurements}

The 2008 measurement [2] is an independent measurement that is consistent with the 2006 measurement [1]. Essentially the same apparatus is used to make a fresh data set. However, the apparatus is now better understood and both the measurement and analysis procedures are significantly improved. For example, the electron is used as a relative magnetometer to allow many data sets, measured on different days, to be combined. This gives lineshape curves with a signal-to-noise good enough to compare their shape with theoretical expectations. (Previously, each day's measurements were combined to get a $g$ value, and the values from different days were averaged.)

The biggest reduction in the uncertainty in our second measurement comes from a better understanding of cavity shifts. Two independent probes of the cavity-mode structure allow identification of nearly all modes and the quantification of an offset between the effective center of the trap for the radiation modes and for the electrostatic quadrupole potential. By measuring $g / 2$ at four magnetic fields with cavity shifts spanning over 30 times our final uncertainty, we precisely test this once-dominant uncertainty, and demonstrate that we can assign an uncertainty that is much smaller than that estimated for our first measurement.

Retroactively applying the improved understanding and the modified analysis developed for the second measurement to the first would require starting from the raw data. Improvements in measurement methods cannot be retroactively implemented, of course. We thus believe that the 2008 measurement should be regarded as superseding the 2006 measurement rather than trying to average the two measurements, which would only insignificantly change the value of $g / 2$ in the second digit of the 2008 uncertainty. The correlations between the possible systematic uncertainties that limit the two measurements has neither been studied nor reported carefully enough to allow an appropriate averaging of the two measurements.

\section{B. Most accurate determination of $\alpha$}

The new measurements of the electron $g / 2$ determine the fine structure constant about 12 times more accurately than the next-most-precise method (Fig. 2). The relationship between $g / 2$ and $\alpha$ has been summarized in detail in [23], with the final value updated in [2]. Here we give only the results and a brief summary.

Within the standard model of particle physics the electron $g / 2$ is related to $\alpha$ by

$$
\begin{aligned}
\frac{g}{2}= & 1+C_{2}\left(\frac{\alpha}{\pi}\right)+C_{4}\left(\frac{\alpha}{\pi}\right)^{2}+C_{6}\left(\frac{\alpha}{\pi}\right)^{3}+C_{8}\left(\frac{\alpha}{\pi}\right)^{4} \\
& +\cdots+a_{\mu, \tau}+a_{\text {hadronic }}+a_{\text {weak }}
\end{aligned}
$$

The leading contribution to $g / 2$ is the 1 that is predicted for a Dirac point particle. Vacuum fluctuations modify the interaction of the electron with the magnetic field, increasing the effective magnetic moment of the electron by approximately one part per thousand. This addition is described by the infinite QED series in powers of $\alpha / \pi$, with coefficients $C_{n}$ determined by $n$-vertex QED calculations for the interaction of electrons and photons. The first three coefficients $\left(C_{2}, C_{4}\right.$, and $C_{6}$ ) have all been calculated exactly. A substantial numerical calculation has determined $C_{8}$, and a numerical calculation of $C_{10}$ is underway. A related series involving the $\mu$ and $\tau$ leptons yields a small contribution, $a_{\mu, \tau}$. Much smaller hadronic and weak contributions, $a_{\text {hadronic }}$ and $a_{\text {weak }}$, have been calculated accurately enough that they do not add uncertainty at the current level of precision. References to the most recently calculated values are provided in [23].

The fine structure constant is determined from the measured $g / 2$ by solving Eq. (66) for $\alpha$ to obtain

$$
\begin{aligned}
\alpha^{-1} & =137.035999084(33)(39)[0.24 \mathrm{ppb}][0.28 \mathrm{ppb}] \\
& =137.035999084(51)[0.37 \mathrm{ppb}]
\end{aligned}
$$

The first line shows experimental (first) and theoretical (second) uncertainties that are nearly the same. The uncertainty in $\alpha$ is now limited a bit more by the need for a higher-order QED calculation (underway [25]) than by the measurement uncertainty in $g / 2$.

In more detail, the theory uncertainty contribution to $\alpha$ is divided as (12) and (37) for $C_{8}$ and $C_{10}$. It should decrease when a calculation underway [25] replaces the crude estimate $C_{10}=0.0(4.6)[20,23]$. The $\alpha^{-1}$ of Eq. (67) will then shift by $2 \alpha^{3} \pi^{-4} C_{10}$, which is $8.0 C_{10} \times 10^{-9}$. A change $\Delta_{8}$ in the calculated $C_{8}=-1.9144(35)$ would add $2 \alpha^{2} \pi^{-3} \Delta_{8}$.

The independent methods for determining $\alpha$ that come closet to our accuracy are the "atom-recoil" measurements, so called because their uncertainty is limited by measurements of recoil velocities in $\mathrm{Rb}$ and $\mathrm{Cs}$ atoms. They rely on many experiments, including the measured Rydberg constant [58,59], the Rb or Cs mass in amu [60], and the electron mass in amu $[61,62]$. The needed $h / M[\mathrm{Rb}]$ comes from a measurement of the recoil of a $\mathrm{Rb}$ atom in an optical lattice $[26,28,63]$. The needed $h / M[\mathrm{Cs}$ ] comes from an optical measurement of the Cs D1 line [27] and the "preliminary" recoil shift for a Cs atom in an atom interferometer [64]. Although these determinations of $\alpha$ have an uncertainty that is currently 12-22 times larger 
than ours, improvements are expected in experiments that are underway $[65,66]$.

\section{Most precise test of QED}

The most stringent test of QED, to the highest order in $\alpha / \pi$, comes from comparing the measured $g / 2$ to the value calculated using Eq. (66) using the best available value of $\alpha$ that is not determined from the electron $g$. Our latest $g$, compared to Eq. (66) with $\alpha(\mathrm{Rb})$, gives a difference [23,28]

$$
|\delta g / 2|<5 \times 10^{-12} .
$$

The good agreement testifies to the remarkable success of QED. The prototype of modern physics theories is thus tested far more stringently than its inventors ever envisioned [67].

The latest $g / 2$ measurement is now accurate enough to allow a 10-20 times more stringent test of QED, should a comparable-accuracy measurement of $\alpha$ become available. We thus strongly emphasize the compelling need for greatly improved independent measurements of $\alpha$.

\section{Limits on electron substructure}

The same comparison of the measured $g / 2$ and the value calculated from Eq. (66) using the best available independent $\alpha$ probes the internal structure of the electron $[23,68]$. A composite electron is constrained to have constituents with a mass $m^{*}>m / \sqrt{\delta g / 2}=230 \mathrm{GeV} / c^{2}$, corresponding to an electron radius $R<1 \times 10^{-18} \mathrm{~m}$.

If this test was limited only by the experimental uncertainty in $g / 2$ (i.e., if a much better independent $\alpha$ becomes available) then we could set a limit $m^{*}>1 \mathrm{TeV}$. These high energy limits seem somewhat remarkable for an experiment carried out at $100 \mathrm{mK}$. However, a search for a contact interaction in electron-positron collisions at LEP sets a more stringent limit, $m^{*}>10 \mathrm{TeV}$ [69].

\section{E. Test of CPT invariance with leptons}

Already the most precise test of CPT invariance with a lepton system comes from comparing the measured magnetic moment of the positron and the electron [4]. A new measurement underway at Harvard aims to improve the sensitivity of this test by a factor of 15 or more, by applying the demonstrated new electron methods to a positron.

\section{F. Application to dark matter}

The comparison of the measured $g / 2$ and the value calculated from Eq. (66) using the best available independent $\alpha$ is also relevant to one model that attempts to explain dark matter. The measured $g / 2$ is accurate enough to allow the discovery of, or to rule out, proposed dark-matter particles with a mass that is close to the electron mass [70], if and when a more accurate independent measurement of $\alpha$ becomes available.

\section{OUTLOOK}

The new $g / 2$ prepares the way for further tests of the standard model, pending the availability of an independent $\alpha$ at the uncertainty reported here. In addition, the techniques used to measure the electron $g / 2$ clear the way for a series of new measurements, some of which have already begun.

First, measuring the positron $g / 2$ to the same precision would improve upon the most stringent lepton CPT test [4] and constrain possible violations of Lorentz invariance [71]. Except for the loading mechanism and an inverted ring voltage, a positron $g / 2$ measurement would proceed identically to the electron measurement presented here.

Second, a direct measurement of the proton-to-electron mass ratio would combine the sub-ppb electron cyclotron frequency resolution presented here with existing techniques for $90 \mathrm{ppt}$ resolution of the proton cyclotron frequency [72] to compete with the existing $0.4 \mathrm{ppb}$ limit [21].

Third, recent observations with a single trapped proton [73] open the way to proposed direct measurements of the proton and antiproton magnetic moments at the ppb scale [11,74]. These would reduce the existing uncertainties by factors of 10 and $10^{6}$, respectively, and provide an important test of CPT invariance [75]. The challenge to such a measurement is QND detection of a spin flip in a magnetic bottle because the smaller magnetic moment and larger mass reduce the axial frequency shift by over $10^{4}$, though a larger bottle gradient can compensate for some of the reduction. Our goal is to realize with a proton and antiproton the great signal-to-noise ratio and sensitivity to frequency changes that have been realized with the one-electron SEO.

Fourth, access to the lowest quantum states of a trapped electron and the lack of radiative damping of any degree of freedom except cyclotron motion have led to several quantum information proposals using electrons in Penning traps, e.g., [76-79] that perhaps could be realized in a carefully optimized planar Penning trap [80].

\section{CONCLUSION}

In conclusion, precise control of the location of and the coupling to the electromagnetic modes of the electrode cavity reduces the once-dominant cavity-shift uncertainty. This results in a measurement of the electron magnetic moment 15 times more accurate than the 1987 measurement that provided the best $g / 2$ and $\alpha$ for nearly 20 years. With the measurement limited by the resolution and model of the cyclotron and anomaly lines, future work on the electron $g / 2$ should focus on enhancing magnetic field stability, narrowing the lines, and building signal-to-noise. The techniques used in this result may be directly applied in measuring the positron $g / 2$ and may be adapted to a direct proton-to-electron mass ratio, to measurements of the proton and antiproton magnetic moments, and to trapped-electron quantum information studies. With an independent $\alpha$ of similar precision, the new $g / 2$ would make possible 10-times more stringent tests of extensions to standard model.

Note added in proof. An independent measurement of $h / M[\mathrm{Rb}]$ [81] was reported recently. This measurement (along with the measured Rydberg constant, a transition frequency measurement and measured mass ratios) determines $\alpha^{-1}=$ $137.035999037(91)$ with an uncertainty reduced to 1.8 times that of our determination. The remarkable agreement of the two determinations stringently tests QED and verifies the 
muonic and hadronic contributions to $g / 2$. The independent $\alpha$ and the standard model calculation discussed above agrees with our our measured $g / 2$ to a precision $|\delta g / 2|<9 \times 10^{-13}$. This limit restricts the electron radius to $R<4 \times 10^{-19} \mathrm{~m}$ and limits potential composite-electron constituent particles to a total mass of $m^{*}>540 \mathrm{GeV} / c^{2}$. The limit also essentially rules out dark-matter particles with a mass close to the electron mass.

\section{ACKNOWLEDGMENTS}

These measurements built upon substantial earlier experimental contributions of J. Tan, C.H. Tseng, K. Abdullah, D. Enzer, S. Peil, B. D'Urso, and B. Odom. We thank J. Dorr for useful comments on the manuscript. This work was supported by the NSF AMO division.
[1] B. Odom, D. Hanneke, B. D’Urso, and G. Gabrielse, Phys. Rev. Lett. 97, 030801 (2006).

[2] D. Hanneke, S. Fogwell, and G. Gabrielse, Phys. Rev. Lett. 100, 120801 (2008)

[3] S. Peil and G. Gabrielse, Phys. Rev. Lett. 83, 1287 (1999).

[4] R. S. Van Dyck Jr., P. B. Schwinberg, and H. G. Dehmelt, Phys. Rev. Lett. 59, 26 (1987).

[5] G. W. Bennett et al., Phys. Rev. D 73, 072003 (2006).

[6] G. Gabrielse and F. C. MacKintosh, Int. J. Mass Spectrom. Ion Processes 57, 1 (1984).

[7] J. N. Tan and G. Gabrielse, Appl. Phys. Lett. 55, 2144 (1989).

[8] J. Tan and G. Gabrielse, Phys. Rev. Lett. 67, 3090 (1991).

[9] J. N. Tan and G. Gabrielse, Phys. Rev. A 48, 3105 (1993).

[10] G. Gabrielse, J. N. Tan, and L. S. Brown, "Cavity shifts of measured electron magnetic moments," in Quantum Electrodynamics, edited by T. Kinoshita (World Scientific, Singapore, 1990), pp. 389-418.

[11] B. D’Urso, R. Van Handel, B. Odom, D. Hanneke, and G. Gabrielse, Phys. Rev. Lett. 94, 113002 (2005).

[12] D. Hanneke, Ph.D. thesis, Harvard University, 2007.

[13] A. Rich and J. C. Wesley, Rev. Mod. Phys. 44, 250 (1972).

[14] G. Graeff, F. G. Major, R. W. T. Roeder, and G. Werth, Phys. Rev. Lett. 21, 340 (1968).

[15] H. Dehmelt and F. Walls, Phys. Rev. Lett. 21, 127 (1968).

[16] G. Graeff, E. Klempt, and G. Werth, Z. Phys. 222, 201 (1969).

[17] F. Walls and T. Stein, Phys. Rev. Lett. 31, 975 (1973).

[18] D. Wineland, P. Ekstrom, and H. Dehmelt, Phys. Rev. Lett. 31, 1279 (1973).

[19] R. S. Van Dyck Jr., P. B. Schwinberg, and H. G. Dehmelt, Phys. Rev. Lett. 38, 310 (1977).

[20] P. J. Mohr and B. N. Taylor, Rev. Mod. Phys. 77, 1 (2005).

[21] P. J. Mohr, B. N. Taylor, and D. B. Newall, Rev. Mod. Phys. 80, 633 (2008).

[22] I. M. Mills, P. J. Mohr, T. J. Quinn, B. N. Taylor, and E. R. Williams, Metrologia 43, 227 (2006).

[23] G. Gabrielse, D. Hanneke, T. Kinoshita, M. Nio, and B. Odom, Phys. Rev. Lett. 97, 030802 (2006); 99, 039902 (2007).

[24] G. Gabrielse, in Lepton Dipole Moments, edited by B. L. Roberts and W. J. Marciano (World Scientific, Singapore, 2009), Chap. 6, pp. 195-218.

[25] T. Aoyama, M. Hayakawa, T. Kinoshita, and M. Nio, Phys. Rev. Lett. 99, 110406 (2007).

[26] P. Cladé, E. de Mirandes, M. Cadoret, S. Guellati-Khélifa, C. Schwob, F. Nez, L. Julien, and F. Biraben, Phys. Rev. Lett. 96, 033001 (2006).

[27] V. Gerginov, K. Calkins, C. E. Tanner, J. J. McFerran, S. Diddams, A. Bartels, and L. Hollberg, Phys. Rev. A 73, 032504 (2006).
[28] M. Cadoret, E. de Mirandes, P. Cladé, S. Guellati-Khélifa, C. Schwob, F. Nez, L. Julien, and F. Biraben, Phys. Rev. Lett. 101, 230801 (2008).

[29] L. S. Brown and G. Gabrielse, Rev. Mod. Phys. 58, 233 (1986).

[30] R. S. Van Dyck Jr., P. B. Schwinberg, and H. G. Dehmelt, The Electron (Kluwer Academic Publishers, Netherlands, 1991), pp. 239-293.

[31] L. S. Brown and G. Gabrielse, Phys. Rev. A 25, 2423 (1982).

[32] L. S. Brown, G. Gabrielse, J. N. Tan, and K. C. D. Chan, Phys. Rev. A 37, 4163 (1988).

[33] G. Gabrielse, Phys. Rev. A 27, 2277 (1983).

[34] J. D. Jackson, Classical Electrodynamics, 3rd ed. (John Wiley \& Sons, New York, 1999).

[35] G. Gabrielse, X. Fei, L. A. Orozco, R. L. Tjoelker, J. Haas, H. Kalinowsky, T. A. Trainor, and W. Kells, Phys. Rev. Lett. 65, 1317 (1990).

[36] G. Gabrielse and J. Tan, J. Appl. Phys. 63, 5143 (1988).

[37] G. Gabrielse, J. Tan, P. Clateman, L. A. Orozco, S. L. Rolston, C. H. Tseng, and R. L. Tjoelker, J. Magn. Reson. 91, 564 (1991).

[38] E. S. Meyer, I. F. Silvera, and B. L. Brandt, Rev. Sci. Instrum. 60, 2964 (1989).

[39] D. F. Phillips, Ph.D. thesis, Harvard University, 1996.

[40] R. S. Van Dyck Jr., D. L. Farnham, S. L. Zafonte, and P. B. Schwinberg, Rev. Sci. Instrum. 70, 1665 (1999).

[41] B. Odom, Ph.D. thesis, Harvard University, 2004.

[42] G. Gabrielse and H. Dehmelt, Phys. Rev. Lett. 55, 67 (1985).

[43] V. B. Braginsky and F. Y. Khalili, Rev. Mod. Phys. 68, 1 (1996).

[44] B. D'Urso, Ph.D. thesis, Harvard University, 2003.

[45] R. Van Dyck Jr., P. Ekstrom, and H. Dehmelt, Nature (London) 262, 776 (1976).

[46] D. J. Wineland and H. G. Dehmelt, J. Appl. Phys. 46, 919 (1975).

[47] R. Kubo, Rep. Prog. Phys. 29, 255 (1966).

[48] B. D'Urso, B. Odom, and G. Gabrielse, Phys. Rev. Lett. 90, 043001 (2003).

[49] E. M. Purcell, Phys. Rev. 69, 681 (1946).

[50] D. Kleppner, Phys. Rev. Lett. 47, 233 (1981).

[51] F. L. Palmer, Phys. Rev. A 47, 2610 (1993).

[52] L. S. Brown, Ann. Phys. (NY) 159, 62 (1985).

[53] L. S. Brown, G. Gabrielse, K. Helmerson, and J. Tan, Phys. Rev. Lett. 55, 44 (1985).

[54] L. S. Brown, G. Gabrielse, K. Helmerson, and J. N. Tan, Phys. Rev. A 32, 3204 (1985).

[55] L. J. Lapidus, D. Enzer, and G. Gabrielse, Phys. Rev. Lett. 83, 899 (1999).

[56] J. H. Malmberg and T. M. O’Neil, Phys. Rev. Lett. 39, 1333 (1977).

[57] S. Ichimaru, H. Iyetomi, and S. Tanaka, Phys. Rep. 149, 91 (1987). 
[58] M. Niering et al., Phys. Rev. Lett. 84, 5496 (2000).

[59] U. D. Jentschura, S. Kotochigova, E.-O. Le Bigot, P. J. Mohr, and B. N. Taylor, Phys. Rev. Lett. 95, 163003 (2005).

[60] M. P. Bradley, J. V. Porto, S. Rainville, J. K. Thompson, and D. E. Pritchard, Phys. Rev. Lett. 83, 4510 (1999).

[61] D. L. Farnham, R. S. Van Dyck Jr., and P. B. Schwinberg, Phys. Rev. Lett. 75, 3598 (1995).

[62] T. Beier, H. Häffner, N. Hermanspahn, S. G. Karshenboim, H.-J. Kluge, W. Quint, S. Stahl, J. Verdú, and G. Werth, Phys. Rev. Lett. 88, 011603 (2001).

[63] P. Cladé, E. de Mirandes, M. Cadoret, S. Guellati-Khélifa, C. Schwob, F. Nez, L. Julien, and F. Biraben, Phys. Rev. A 74, 052109 (2006).

[64] A. Wicht, J. M. Hensley, E. Sarajlic, and S. Chu, Phys. Scr. T 102, 82 (2002).

[65] H. Müller, S. -W. Chiow, Q. Long, C. Vo, and S. Chu, Appl. Phys. B 84, 633 (2006).

[66] P. Cladé, S. Guellati-Khélifa, F. Nez, and F. Biraben, Phys. Rev. Lett. 102, 240402 (2009).

[67] F. Dyson, "Letter to G. Gabrielse," quoted in Physics Today, August 2006, pp. 15-17.

[68] S. J. Brodsky and S. D. Drell, Phys. Rev. D 22, 2236 (1980).

[69] D. Bourilkov, Phys. Rev. D 64, 071701(R) (2001).

[70] C. Bœhm and J. Silk, Phys. Lett. B 661, 287 (2008).
[71] R. Bluhm, V. A. Kostelecký, and N. Russell, Phys. Rev. Lett. 79, 1432 (1997).

[72] G. Gabrielse, A. Khabbaz, D. S. Hall, C. Heimann, H. Kalinowsky, and W. Jhe, Phys. Rev. Lett. 82, 3198 (1999).

[73] N. Guise, J. DiSciacca, and G. Gabrielse, Phys. Rev. Lett. 104, 143001 (2010).

[74] W. Quint, J. Alonso, S. Djekić, H.-J. Kluge, S. Stahl, T. Valenzuela, J. Verdú, M. Vogel, and G. Werth, Nucl. Instrum. Methods Phys. Res. B 214, 207 (2004).

[75] R. Bluhm, V. A. Kostelecký, and N. Russell, Phys. Rev. D 57, 3932 (1998).

[76] S. Mancini, A. M. Martins, and P. Tombesi, Phys. Rev. A 61, 012303 (1999).

[77] G. Ciaramicoli, I. Marzoli, and P. Tombesi, Phys. Rev. A 63, 052307 (2001).

[78] G. Ciaramicoli, I. Marzoli, and P. Tombesi, Phys. Rev. Lett. 91, 017901 (2003)

[79] G. Ciaramicoli, F. Galve, I. Marzoli, and P. Tombesi, Phys. Rev. A 72, 042323 (2005).

[80] J. Goldman and G. Gabrielse, Phys. Rev. A 81, 052335 (2010).

[81] R. Bouchendira, P. Cladé, S. Guellati-Khélifa, F. Nez, and F. Biraben, Phys. Rev. Lett.106, 080801 (2011). 